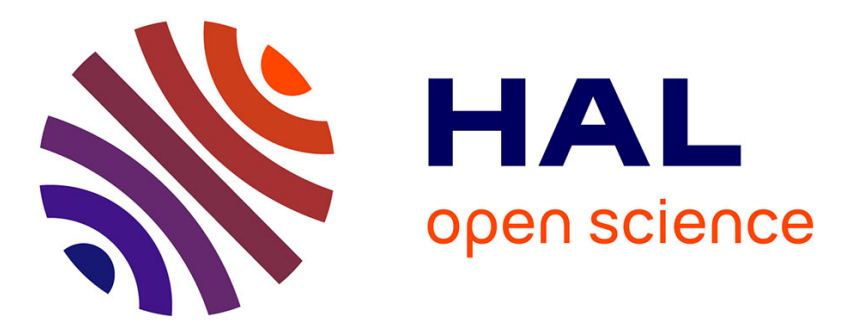

\title{
Constrained state estimation for nonlinear systems: a redesign approach based on convexity
}

Daniele Astolfi, Pauline Bernard, Romain Postoyan, Lorenzo Marconi

\section{To cite this version:}

Daniele Astolfi, Pauline Bernard, Romain Postoyan, Lorenzo Marconi. Constrained state estimation for nonlinear systems: a redesign approach based on convexity. IEEE Transactions on Automatic Control, 2021, 67 (2), pp.824-839. 10.1109/TAC.2021.3064537 . hal-03157454

\section{HAL Id: hal-03157454 https://hal.science/hal-03157454}

Submitted on 3 Mar 2021

HAL is a multi-disciplinary open access archive for the deposit and dissemination of scientific research documents, whether they are published or not. The documents may come from teaching and research institutions in France or abroad, or from public or private research centers.
L'archive ouverte pluridisciplinaire HAL, est destinée au dépôt et à la diffusion de documents scientifiques de niveau recherche, publiés ou non, émanant des établissements d'enseignement et de recherche français ou étrangers, des laboratoires publics ou privés. 


\title{
Constrained state estimation for nonlinear systems: a redesign approach based on convexity
}

\author{
Daniele Astolfi, Pauline Bernard, Romain Postoyan and Lorenzo Marconi
}

\begin{abstract}
Given a plant whose trajectories of interest remain in a known compact set and an associated observer, we propose a general framework to modify this observer so that its state remains in a given convex set for all times, without altering the observer guaranteed performances in terms of convergence and robustness to external disturbances. The methodology can be applied to any time-varying continuous-time, discrete-time and hybrid system/observer, for which a quadratic Lyapunov function is used for the analysis. The proposed approach is relevant, for instance, to remove the peaking phenomenon, to attenuate the effect of impulsive outliers in the measurement, to avoid aberrant estimates during transients, or to guarantee a given range for variables in embedded systems.
\end{abstract}

Index Terms-Nonlinear observers, Lyapunov methods, hybrid dynamical systems, discrete-time, nonlinear systems.

\section{INTRODUCTION}

\section{A. Motivation}

Numerous observer design methods are available in the literature for continuous-time systems, see, e.g., [1]-[14], discrete-time systems, see, e.g. [1], [15]-[21], and hybrid systems [22], see, e.g. [23]-[36], to mention a few. In general, the robust convergence of the state estimate to the plant state is ensured, under suitable conditions. Now, in many applications, the state of the plant is evolving in a given compact set $\mathcal{X}$ describing the set of interest of the variables. However, unless the observation problem is formulated as a constrained optimization problem from the start [37], the estimated state generated by the observer could in principle leave the set $\mathcal{X}$ for some time, because of transient dynamics or perturbations. These excursions outside $\mathcal{X}$ may be significant in terms of amplitude and time, and may lead to the next issues.

- (Accuracy) When the estimate is outside $\mathcal{X}$, the information provided by the observer is likely to be inaccurate and so potentially unexploitable by the user. A typical example is the peaking phenomenon occurring in high-gain observers [7].

D. Astolfi is with Univ Lyon, Université Claude Bernard Lyon 1, CNRS, LAGEPP UMR 5007, 43 Boulevard du 11 Novembre 1918, F-69100, Villeurbanne, France (daniele.astolfi@univ-lyon1.fr).

P. Bernard is with the Systems and Control Center of MINES ParisTech, PSL Research University, 60 boulevard Saint-Michel, 75006 Paris, France (pauline.bernard@mines-paristech.fr)

R. Postoyan is with Université de Lorraine, CNRS, CRAN, F-54000 Nancy, France (romain.postoyan@univ-lorraine.fr).

L. Marconi is with CASY-DEI, University of Bologna, Viale Pepoli 3/2, 40123 Bologna, Italy (lorenzo.marconi@unibo.it).

This research was partially supported by the French Grant ANR ODISSE (ANR-19-CE48-0004-01).
- (Aberrant estimates) The state estimate may violate the physics of the plant. To give an example, when the concentrations of Lithium-ion in the electrodes of an electrochemical battery are estimated, e.g. [38], it may occur that the estimated concentrations generated by the observer are negative for some time, which is physically impossible. This is the case in general for positive systems (see, e.g., [39]).

- (Implementation issues) When implementing an observer on an embedded systems, we often need to specify the range of the estimated states, and this range may be limited.

- (Destabilization) It may lead to instability in output feedback designs, see e.g. [7].

- (Existence of observer dynamics) The observer dynamics may not be well defined outside the set $\mathcal{X}$. This issue is typical when the observer is not implemented in the same coordinates of the plant dynamics, see, e.g., [40]-[43].

A simple idea to address some of these issues is to saturate or to project into $\mathcal{X}$ the state estimate generated by the observer, while preserving its inner dynamics, as done in the output feedback stabilization context [7], [40] or in the context of discrete-time Kalman filtering [44]. This solution, however, does not address some of the previous problems (implementation issues and existence of observer dynamics) as the inner observer state can still venture outside $\mathcal{X}$. A natural way to overcome these issues is to constrain the observer state in a given set $\widehat{\mathcal{X}} \supset \mathcal{X}$. This has nevertheless to be done with care to preserve the observer convergence and performance. In control problems, combining convergence with invariance constraints is typically handled through control barrier functions [45], leading to the so-called safety control. However, those methods do not transpose easily to estimation field because the state, and thus the value of the Lyapunov function, are unknown. Instead, several projection-based approaches have been developed for specific classes of systems and observer designs. In the context of continuous-time systems, projectionbased solutions have been proposed for high-gain observers in [40], for Lur'e type systems in [46], and within the framework of projected dynamical systems in [47] for exponentially convergent observers with linear correction terms. Projection methods are also very well-known in the context of constrained Kalman filters (KF) for discrete-time systems with the socalled equality constrained $\mathrm{KF}$ (ecKF), which was proved to provide a smaller state error covariance than the unconstrained algorithm. An alternative method named system projection approach (spKF) is to implement the unconstrained KF on the projected linear system through a model reduction as proposed in [48]. More generally, nonlinear constraints have also been 
considered as extra measurements [49], but mainly treated via linearization [37], [44], [49], or by taking the first two orders of the Taylor development around the estimate leading to quadratic equality constraints in [50]. A surveys of all those methods (including inequality constraints) is available in [51]. More recently, the case of time-varying linear equality constraints was also considered in [52] for a continuous-time Kalman filter. Those techniques are however not easy to extend to other classes of observers or of constraints (nonlinear, inequality) and can be hard to implement on-line in presence of limited computational resources.

\section{B. Contributions}

To the best of these authors' knowledge, there does not exist a generic solution addressing the problem of constraining the observer dynamics in a given compact set for continuoustime (CT in short), discrete-time (DT) or hybrid systems. All the designs proposed in literature are developed ad-hoc for specific classes of observers. In this context, the objective of this paper is to present a general methodology, that addresses the problem of constraining the state of a given observer into a prescribed compact set $\widehat{\mathcal{X}}$, strictly containing $\mathcal{X}$, for all times, and preserving, at the same time, the guaranteed convergence or performance of the observer inside $\widehat{\mathcal{X}}$. To characterize the performances of the observer, we assume the knowledge of a (possibly time-varying) quadratic Lyapunov function for the estimation error, whose evolution along the solutions to the estimation error system is bounded by a given supply rate. Hence, the performances are quantified by the supply rate associated to the considered Lyapunov function. Our goal is to modify the observer such that those bounds are not changed - we can then say that the guaranteed performances are preserved - while ensuring that the internal state of the observer remains in the given compact set $\widehat{\mathcal{X}}$. For instance, if the derivative of the Lyapunov function along trajectories is bounded by a negative function, such negativity is preserved.

The main ideas of this paper stem from [41], [42] where a gradient redesign approach, based on a convexity assumption, is used for continuous-time high-gain observers implemented in coordinates which are different with respect to those of the plant's dynamics. Following the same spirit, this approach was then adapted to the discrete-time case in our preliminary work [53] by adding fictitious jumps in-between each (sampling) discrete times. The methodology in [53] has been then used in [43] for a specific problem of coordinate-implementation issues in continuous-time observer design.

In this paper, we generalize those constructions by presenting a unifying framework covering a wider class of observer design techniques. To this end, two different tools are developed.

- Flow redesign. The idea is to modify the observer flow map by adding an extra term that makes the chosen set $\widehat{\mathcal{X}} \supset \mathcal{X}$ invariant while preserving the given bounds on the timederivative of the initial Lyapunov function along the system and the modified observer solutions.

- Jump redesign. We compose the observer jump map with a map that does not increase the Lyapunov function at jumps and whose output is in $\widehat{\mathcal{X}} \supset \mathcal{X}$.
With the proposed tools, we are therefore able to address the desired constrained problem in the following cases.

- CT plant with CT observer and flow redesign, resulting in a CT redesigned observer.

- CT plant with CT/hybrid observer and jump redesign, resulting in a hybrid redesigned observer.

- DT plant with DT observer and jump redesign.

- Hybrid plant with hybrid observer and flow and/or jump redesign.

With respect to [41]-[43], we cover a larger class of CT nonlinear observers (e.g., [4]-[6]) including design with timevarying gains [1]-[3]. With respect to [53], we allow the Lyapunov functions to be time-dependent, thus enlarging the classes of discrete-time observers to which the proposed techniques can be applied, such as Kalman-like observers [1]. Moreover, a jump redesign based on a single step is proposed and the robustness of the initial multi-step strategy from [53] is shown based on a set-valued regularization. Finally, the new case of hybrid systems is addressed.

The rest of the paper is organized as follows. We formalize the problem by stating the standing assumptions in Section II. The flow redesign tool is developed in Section III for continuous-time systems with continuous-time observers. Then, the jump redesign tool is presented in Section IV for discrete-time systems with discrete-time observers. Finally, both tools are combined in Section $\mathrm{V}$ to address hybrid observers. In Section VI, we discuss the choice of some parameters characterizing the proposed redesign framework. Simulation results are presented in Section VII and conclusions are drawn in Section VIII.

Notation. $\mathbb{R}, \mathbb{R}_{\geq 0}$ and $\mathbb{N}$ denote the set of real numbers, nonnegative real numbers and positive integer numbers, respectively. We define $\mathbb{R}_{\geq 0}^{n}:=\mathbb{R}_{\geq 0} \times \cdots \times \mathbb{R}_{\geq 0}$. We denote with $|\cdot|$ the standard Euclidean norm. We say that a function $f: X \rightarrow \mathbb{R}$ is bounded if $\sup _{x \in X}|f(x)|<\infty$. Given to sets $S_{1}, S_{2} \subseteq \mathbb{R}^{n}$, we define the distance from two sets as $d\left(S_{1}, S_{2}\right):=\inf \left\{|x-y|: x \in S_{1}, y \in S_{2}\right\}, S_{1} \subseteq S_{2}$ denotes an inclusion and $S_{1} \subset S_{2}$ denotes a strict inclusion such that $d\left(S_{1}, \mathbb{R}^{n} \backslash S_{2}\right)>0$. For a set-valued map $V: \mathbb{R}^{p} \rightrightarrows \mathbb{R}$ and a scalar $c, V(x) \leq c$ means that $v \leq c$ for all $v \in V(x)$. For a set-valued map $\mathcal{F}: \mathbb{R}^{q} \rightrightarrows \mathbb{R}^{p}, V: \mathbb{R}^{p} \rightarrow \mathbb{R}$, and $x \in \mathbb{R}^{q}, L_{\mathcal{F}} V(x)=\sup \left\{L_{v} V(x): v \in \mathcal{F}(x)\right\}$, where $L_{v} V(x):=\frac{\partial V}{\partial x}(x) v$ is the Lie derivative of $V$ along the vector $v$ at the point $x$. We refer to the definition of outer semicontinuity given in [22, Definition 5.9]. We refer to standard notations of class- $\mathcal{K}$ and class- $\mathcal{K} \mathcal{L}$ functions, see, i.e. $[22$, Chapter 3].

\section{STANDING ASSUMPTIONS AND MAIN IDEA}

We consider a plant $\mathcal{P}$ modelled as a finite-dimensional time-varying dynamical system with state $x \in \mathbb{R}^{n_{x}}$, that is either continuous-time, discrete-time or hybrid, with measured outputs $y \in \mathbb{R}^{n_{y}}$ and known inputs $u \in \mathbb{R}^{n_{u}}$, and is affected by unknown perturbations $d \in \mathbb{R}^{n_{d}}$, with $n_{x}, n_{u}, n_{y}, n_{d} \in \mathbb{N}$, as formalized in the next sections.

Standing Assumption 1 (SA1) There exist subsets $\mathcal{X}_{0} \subset$ $\mathbb{R}^{n_{x}}, \mathcal{U} \subset \mathbb{R}^{n_{u}}, \mathcal{D} \subset \mathbb{R}^{n_{d}}$, and a compact subset $\mathcal{X} \subset \mathbb{R}^{n_{x}}$, 
such that any trajectory of $\mathcal{P}$ initialized in $\mathcal{X}_{0}$, with input in $\mathcal{U}$ and perturbation in $\mathcal{D}$, remains in $\mathcal{X}$ for all forward times.

No restriction is made on the "size" of the sets in SA1, these can therefore be arbitrarily large. Actually, only the knowledge of $\mathcal{X}$ is necessary for design. In other words, we assume the plant trajectories to be estimated evolve in a known compact set $\mathcal{X}$, which is not required to be invariant for the dynamics. Although this assumption could seem like a limitation of this framework, boundedness of solutions is commonly verified in practical applications, where the state variables satisfy known physical bounds. Finally, note that such a boundedness property does not necessarily mean the plant model exhibits stability properties.

We then assume that we know an observer $\mathcal{O}$ for plant $\mathcal{P}$ with some guaranteed properties, as formalized in the next sections, whose state estimate is denoted $\hat{x} \in \mathbb{R}^{n_{x}}$. At this stage, any existing observer can be considered; detailed examples are discussed in the sequel. While the state $x$ of plant $\mathcal{P}$ is known to lie in $\mathcal{X}$, according to $\mathrm{SA} 1$, it may occur that the state $\hat{x}$ of observer $\mathcal{O}$ leaves $\mathcal{X}$ for some time, as explained in the introduction. Therefore, given a prescribed compact set $\widehat{\mathcal{X}} \subset \mathbb{R}^{n}$, with $\widehat{\mathcal{X}} \supset \mathcal{X}$, the goal of the paper is to redesign observer $\mathcal{O}$ so that the following holds.

1) The modified observer, denoted as $\mathcal{O}_{m}$, exhibits the same guaranteed performances as the original observer.

2) The state of the redesigned observer $\mathcal{O}_{m}$ remains in $\widehat{\mathcal{X}}$ for all forward times when initialized in $\mathcal{X}$.

As it is often the case in designs with constraints (see, e.g., [40], [42], [47], [54]), the solutions presented in the sequel rely on a key convexity assumption, as formalized next.

Standing Assumption 2 (SA2) The convex hull of $\mathcal{X}$ is strictly included in $\widehat{\mathcal{X}}$, i.e., there exists a compact convex set $\mathcal{C} \in \mathbb{R}^{n_{x}}$ satisfying $\mathcal{X} \subseteq \mathcal{C} \subset \widehat{\mathcal{X}}$.

This assumption says that it will not be possible with our approach to keep the observer state in any non-convex set $\mathcal{X}$, but only in a compact convex set containing $\mathcal{X}$. For instance, our approach cannot be used to keep the observer state away from singularities, or close to a non-convex manifold. Although this assumption may seem restrictive, it actually enables to keep the estimate within polytopes and hyperspheres modelling known physical bounds on the plant state, which is of significant practical interest. A more detailed discussion about the choice of $\widehat{\mathcal{X}}$ is provided in Section VI, after presenting the main results of this work.

SA2 implies the following convexity result (see an illustration in Figure 1), which plays an essential role in the sequel.

Lemma 1 Suppose SA2 holds. Then, there exist $\varrho \in \mathbb{R}_{>0}$, an integer $n_{c} \in \mathbb{N}$, and a $C^{1}$ function $c: \mathbb{R}^{n_{x}} \rightarrow \mathbb{R}_{\geq 0}^{n_{c}}$ such that, by defining

$$
\mathcal{C}_{0}:=\left\{x \in \mathbb{R}^{n_{x}}: c(x)=0\right\}, \mathcal{C}_{\varrho}:=\left\{x \in \mathbb{R}^{n_{x}}:|c(x)| \leq \varrho\right\},
$$

the following holds:

a) $\mathcal{X} \subseteq \mathcal{C}_{0} \subset \mathcal{C}_{\varrho} \subset \widehat{\mathcal{X}}$.

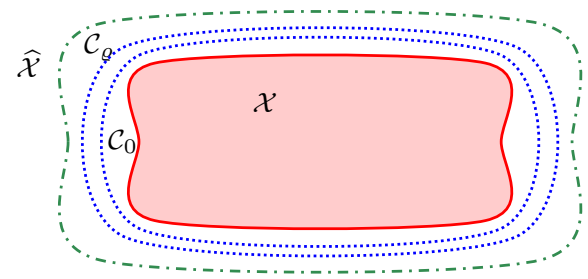

Fig. 1. Illustration of sets $\mathcal{X}$ (in red), $\widehat{\mathcal{X}}$ (in dash-dotted green) and $\mathcal{C}_{0}, \mathcal{C}_{\varrho}$ (in dotted blue) satisfying item a) of Lemma 1 and thus SA2.

b) For any $i=1, \ldots, n_{c}$, the $i$-th component $c_{i}: \mathbb{R}^{n_{x}} \rightarrow$ $\mathbb{R}_{\geq 0}$ of $c$ is convex.

Proof. According to [55, (6)], any compact convex set $\mathcal{C}$ can be outer-approximated as closely as desired by a polytope. Therefore, by continuity, and since $\mathcal{C}$ is strictly included in $\widehat{\mathcal{X}}$, there exist $a_{i} \in \mathbb{R}^{n_{x}}$ and $b_{i} \in \mathbb{R}$ for $i=1, \ldots, n_{c}$, with $n_{c}$ large enough, such that $\mathcal{X} \subseteq \bigcap_{i=1}^{n_{c}}\left\{x \in \mathbb{R}^{n_{x}}: a_{i}^{\top} x \leq\right.$ $\left.b_{i}\right\} \subset \widehat{\mathcal{X}}$. Lemma 7 in Section VI shows how to build a map $c$ verifying items a) and $b$ ).

A key feature of the map $c$ is that it vanishes on $\mathcal{X}$ where the plant trajectories evolve, since $\mathcal{X} \subseteq \mathcal{C}_{0}$. Following [41], the main idea underlying the redesign constructions proposed in this work, is to associate to any trajectory of the plant $\mathcal{P}$ an extra "dummy" output $y_{2}=c(x)$ which is constantly zero, since $x \in \mathcal{X}$, and to observe that the corresponding estimated version $\hat{y}_{2}=c(\hat{x})$ is not zero whenever $\hat{x}$ goes outside $\mathcal{X}$. Therefore, we can modify the observer $\mathcal{O}$ by adding a second output injection term, driven by the measured difference $\hat{y}_{2}-$ $y_{2}$, to correct the trajectories of the observer $\mathcal{O}$ outside the set $\mathcal{C}_{0}$. This will be done by exploiting the next convexity property of $c$.

Lemma 2 Consider a $C^{1}$ function $c: \mathbb{R}^{n_{x}} \rightarrow \mathbb{R}_{\geq 0}^{n_{c}}$ satisfying item $b)$ of Lemma 1. Then, for all $(x, \hat{x}) \in \mathbb{R}^{n_{x}} \times \mathbb{R}^{n_{x}}$ such that $c(x)=0$, we have

$$
-c(\hat{x})^{\top} \frac{d c}{d x}(\hat{x})(\hat{x}-x) \leq-c(\hat{x})^{\top} c(\hat{x}) \leq 0 .
$$

Proof. The convexity of $c_{i}$, for $i=1, \ldots, n_{c}$, implies that $c_{i}(x) \geq c_{i}(\hat{x})+\frac{d c_{i}}{d x}(\hat{x})(x-\hat{x})$ for all $x, \hat{x} \in \mathbb{R}^{n_{x}}$, and therefore that $-\frac{d c_{i}}{d x}(\hat{x})(\hat{x}-x) \leq-c_{i}(\hat{x})$ if $c(x)=0$. Inequality (2) then follows directly by decomposing the scalar product and using the positivity of $c_{i}$.

As a consequence, in the rest of the paper, we suppose the elements of Lemma 1 are known and available for the design. Insights about the way $\widehat{\mathcal{X}}$, the function $c$ and $\varrho$ can be chosen/built are given in Section VI.

\section{The Continuous-Time CASE}

\section{A. Problem Statement}

We assume in this section that the plant $\mathcal{P}$ is described as

$$
\mathcal{P}: \quad \dot{x}=f(t, x, u, d), \quad y=h(t, x, u, d),
$$

with state $x \in \mathbb{R}^{n_{x}}$, known input $u \in \mathbb{R}^{n_{u}}$, output $y \in \mathbb{R}^{n_{y}}$, and where $d \in \mathbb{R}^{n_{d}}$ is an unknown perturbation acting on 
the dynamics and/or the measurement. We consider then a continuous-time observer of the form

$$
\mathcal{O}: \quad \dot{\hat{x}}=F(t, \hat{x}, u, y),
$$

with state $\hat{x} \in \mathbb{R}^{n_{x}}$. System (3) and observer (4) satisfy some regularity properties as stated in the next assumption.

Assumption 1 The following is satisfied:

- The function $h$ in (3) is bounded in its first argument and the set $\mathcal{Y}:=h\left(\mathbb{R}_{\geq 0}, \mathcal{X}, \mathcal{U}, \mathcal{D}\right)$ is compact.

- The function $F$ is bounded on $\mathbb{R}_{\geq 0} \times \widehat{\mathcal{X}} \times \mathcal{U} \times \mathcal{Y}$.

- The solutions of (4) exist forward in time for any initial condition $\hat{x}(0) \in \mathcal{X}$, any input $u$ in $\mathcal{U}$ and any output $y$ of system (4), i.e. no finite-escape time phenomenon is possible.

Furthermore, we assume that the observer (4) has been designed so that the following assumption is verified.

Assumption 2 There exist a (possibly time varying) known matrix $P(t)=P(t)^{\top}>0$, differentiable in $t$, real numbers $\bar{\lambda}>\underline{\lambda}>0$, and a function $\beta_{c}: \mathbb{R}_{\geq 0} \times \mathbb{R}^{n_{x}} \times \mathbb{R}^{n_{x}} \times \mathbb{R}^{n_{u}} \times$ $\mathbb{R}^{n_{d}} \rightarrow \mathbb{R}$, such that, by defining

$$
V(t, x, \hat{x}):=(\hat{x}-x)^{\top} P(t)(\hat{x}-x),
$$

the following is satisfied

$$
\begin{aligned}
& \underline{\lambda}|x-\hat{x}|^{2} \leq V(t, x, \hat{x}) \leq \bar{\lambda}|x-\hat{x}|^{2} \\
& L_{\mathcal{F}} V(t, x, \hat{x}, u, d) \leq \beta_{c}(t, x, \hat{x}, u, d)
\end{aligned}
$$

for all $(t, x, \hat{x}, u, d) \in \mathbb{R}_{\geq 0} \times \mathcal{X} \times \mathbb{R}^{n_{x}} \times \mathcal{U} \times \mathcal{D}$, with

$$
\mathcal{F}(t, x, \hat{x}, u, d):=(1, f(t, x, u, d), F(t, \hat{x}, u, h(t, x, u, d))) .
$$

Assumption 2 characterizes the performances of observer (4) in terms of the function $V$ and the supply rate $\beta_{c}$. For instance, for an exponentially stable observer which is inputto-state stable (ISS) with respect to the perturbation, the function $\beta_{c}$ in (7) is typically of the form

$$
\beta_{c}(t, x, \hat{x}, u, d)=-\rho_{c} V(t, x, \hat{x})+\alpha_{c}(|d|),
$$

with $\rho_{c}>0$ and $\alpha_{c} \in \mathcal{K}$. In this case, $V$ is the Lyapunov function associated to the estimation error $x-\hat{x}$ and $\rho_{c}$ is the convergence rate. Note that, when the matrix $P$ is constant, $\bar{\lambda}$ and $\underline{\lambda}$ in (6) correspond to the maximum and minimum eigenvalues of $P$, respectively.

Assumption 2 covers a large class of observers for the design of $\mathcal{O}$ in (4). For instance, the following observers satisfy the conditions of Assumption 2 with $P$ constant:

- linear Luenberger observers [4], when (3) is linear;

- high-gain nonlinear observers [7], [8] for systems in triangular forms with Lipschitz nonlinearities;

- observer designs based on linear parameter-varying-like techniques or the circle criterion, e.g., [5], [6].

Furthermore, Kalman filters [1] and Kalman-like observers [2], [3] used for state-affine normal forms, also satisfy Assumption 2 with a time-varying $P$. In these cases, $P$ is solution to a differential Riccati or Lyapunov equation that is independent from the estimate $\hat{x}$ and uniformly upper and lower bounded for all positive times. These extra dynamics are implemented online and $P$ is considered as an external time-varying input for observer (4). See also [14, Section VI] for further examples.

Note also that in the context of nonlinear observers, one may need to change coordinates before designing an observer based on a quadratic Lyapunov function (nonlinear Luenberger design [9], high-gain design [56]). In that case, the methods developed in this paper should be applied in those new coordinates, as done for instance in [42], [43].

The objective of this section is to modify observer (4) in order to solve the next problem.

Problem 1 Design a map $F_{m}: \mathbb{R} \times \mathbb{R}^{n_{x}} \times \mathbb{R}^{n_{u}} \times \mathbb{R}^{n_{y}}$ such that the following holds.

1) For any initial condition $(x(0), \hat{x}(0)) \in \mathcal{X}_{0} \times \mathcal{X}$, any input in $\mathcal{U}$ and perturbation in $\mathcal{D}$, the corresponding trajectory of the modified observer

$$
\mathcal{O}_{m}: \quad \dot{\hat{x}}=F_{m}(t, \hat{x}, u, y)
$$

remains in $\widehat{\mathcal{X}}$ for all $t \geq 0$.

2) The Lyapunov function (5) satisfies

$$
L_{\mathcal{F}_{m}} V(t, x, \hat{x}, u, d) \leq \beta_{c}(t, x, \hat{x}, u, d)
$$

for any $(t, x, \hat{x}, u, d) \in \mathbb{R}_{\geq 0} \times \mathcal{X} \times \widehat{\mathcal{X}} \times \mathcal{U} \times \mathcal{D}$, with $\beta_{c}$ coming from Assumption 2 and

$$
\begin{aligned}
& \mathcal{F}_{m}(t, x, \hat{x}, u, d):= \\
& \left(1, f(t, x, u, d), F_{m}(t, \hat{x}, u, h(t, x, u, d))\right) .
\end{aligned}
$$

Hence, by solving Problem 1, the state estimate generated by observer $\mathcal{O}_{m}$ remains in set $\widehat{\mathcal{X}}$ and property (7) is preserved for $\mathcal{O}_{m}$ as desired.

\section{B. Observer Redesign}

We modify the dynamics of observer (4) as

$$
F_{m}(t, \hat{x}, u, y):=F(t, \hat{x}, u, y)+M(t, \hat{x})
$$

where function $M$ is defined by

$$
M(t, \hat{x}):=-\gamma_{c} P(t)^{-1} \frac{d c}{d x}(\hat{x})^{\top} c(\hat{x})
$$

with $c$ defined in Lemma 1, $P$ given by Assumption 2, and $\gamma_{c} \in \mathbb{R}_{>0}$ a parameter to be chosen large enough. Note that the knowledge of the supply rate $\beta_{c}$ is not needed for the design of (13). In order to state the main result of this section, we define the set $\partial \mathcal{C}_{\varrho}$ and the positive real number $\delta_{\min }$ as

$$
\begin{aligned}
\partial \mathcal{C}_{\varrho} & :=\left\{x \in \mathbb{R}^{n_{x}}:|c(x)|=\varrho\right\}, \\
\delta_{\min } & :=\inf _{x \in \partial \mathcal{C}_{\varrho}}\left|\frac{d c}{d x}(x)^{\top} c(x)\right|,
\end{aligned}
$$

with $c, \varrho$ given in Lemma 1 . We have the following result.

Theorem 1 Suppose Assumptions 1 and 2 hold and that $\delta_{\min }$ in (16) is strictly positive. Then, there exists $\gamma_{c}^{\star} \geq 0$ such that, for any $\gamma_{c}>\gamma_{c}^{\star}$, Problem 1 is solved with $F_{m}$ defined in (13), (14). 
Proof. First, we define $F_{\max }, \gamma_{c, \min }$ as

$$
\begin{aligned}
F_{\max } & :=\sup _{(t, \hat{x}, u, y) \in \mathbb{R}_{\geq 0} \times \partial \mathcal{C}_{\varrho} \times \mathcal{U} \times \mathcal{Y}}|F(t, \hat{x}, u, y)|, \\
\gamma_{c}^{\star} & :=\frac{F_{\max } \bar{\lambda}}{\delta_{\min }},
\end{aligned}
$$

with $\mathcal{Y}$ defined in Assumption 1, and $\bar{\lambda}$ given by Assumption 2. In light of the properties of $F$ in Assumption 1, and the fact that $\partial \mathcal{C}_{\varrho} \subset \widehat{\mathcal{X}}$ in view of item (a) of Lemma 1 , the real numbers $F_{\max }, \gamma_{c}^{\star}$ are finite. Now consider the function $W(\hat{x}):=\frac{1}{2} c(\hat{x})^{\top} c(\hat{x})$ for any $\hat{x} \in \mathbb{R}^{n_{x}}$, and pick $\hat{x} \in \partial \mathcal{C}_{\varrho}, u$ in $\mathcal{U}$ and $x$ in $\mathcal{X}$. By using (16), (17), (18), we compute

$$
\begin{aligned}
& \left\langle\nabla W(\hat{x}), F_{m}(t, \hat{x}, u, y)\right\rangle \\
& \quad=c(\hat{x})^{\top} \frac{d c}{d x}(\hat{x})\left[F(t, \hat{x}, u, y)-\gamma_{c} P(t)^{-1} \frac{d c}{d x}(\hat{x})^{\top} c(\hat{x})\right] \\
& \leq\left|\frac{d c}{d x}(\hat{x})^{\top} c(\hat{x})\right|\left(F_{\max }-\frac{\gamma_{c}}{\bar{\lambda}} \delta_{\min }\right)<0
\end{aligned}
$$

in which we used the fact that, in light of Assumption 2, $P(t)$ satisfies $\frac{1}{\bar{\lambda}} I \leq P(t)^{-1}$ for all $t \geq 0$, and $\delta_{\min }>0$. This shows that the derivative of $W$ along solutions to $\mathcal{O}_{m}$ is strictly negative on $\partial \mathcal{C}_{\varrho}$, which implies that $\mathcal{C}_{\varrho}$ is forward invariant. A fortiori, any trajectory initialized in $\mathcal{C}_{\varrho}$ remains in $\widehat{\mathcal{X}}$ as $\mathcal{C}_{\varrho} \subset \widehat{\mathcal{X}}$ according to Lemma 1 and therefore item 1) of Problem 1 is proved since $\mathcal{X} \subset \mathcal{C}_{\varrho} \subset \widehat{\mathcal{X}}$ in view of item (a) of Lemma 1.

By using the definition of $\mathcal{F}_{m}$ and $M$ in (12) and (14), and the definition of $\beta_{c}$ in Assumption 2, we obtain for all $(t, x, \hat{x}, u, d) \in \mathbb{R} \times \mathcal{X} \times \widehat{\mathcal{X}} \times \mathcal{U} \times \mathcal{D}$

$$
\begin{aligned}
L_{\mathcal{F}_{m}} V(t, & x, \hat{x}, u, d) \\
& =L_{\mathcal{F}} V(t, x, \hat{x}, u, d)+2(\hat{x}-x)^{\top} P(t) M(t, \hat{x}) \\
& \leq \beta_{c}(t, x, \hat{x}, u, d)-2 \gamma_{c}(\hat{x}-x)^{\top} \frac{d c}{d x}(\hat{x})^{\top} c(\hat{x}) .
\end{aligned}
$$

By recalling that $c(x)=0$ for any $x \in \mathcal{X}$ by Lemma 1 , and since $\gamma_{c}>0$, we obtain (11) from Lemma 2.

The extra condition $\delta_{\min }>0$ in Theorem 1 is verified for the functions $c$ proposed in Section VI (see (62) and (63) of Lemma 7 and Lemma 8). On the other hand, the role of $\gamma_{c}$ in (14) is to correct the vector field $F$ when the latter becomes too large and makes the estimate leave the set $\mathcal{X}$. To do that, we have chosen a constant gain $\gamma_{c}$ whose magnitude is large enough with respect to the maximal norm of $F$ on the boundary of $\mathcal{C}_{\varrho}$. A bound on $\gamma_{c}$ is given in the proof of Theorem 1, see (18). This bound may be subject to some conservatism and in practice $\gamma_{c}$ can be adapted online to compensate for the "current" value of $F$, by taking for instance

$$
\gamma_{c}(t, \hat{x}, u, y):=\frac{2 \bar{\lambda}|F(t, \hat{x}, u, y)|}{\max \left\{\left|\frac{d c}{d x}(\hat{x})^{\top} c(\hat{x})\right|, \delta_{\min }\right\}}
$$

for any $(t, \hat{x}, u, y) \in \mathbb{R} \times \mathbb{R}^{n_{x}} \times \mathcal{U} \times \mathcal{Y}$, in view of the proof of Theorem 1, see [42].

Remark 1 The correction term $M$ defined in (14) may also be interpreted as an element of the normal cone to the level set of $c$ in the metric defined by $P(t)$, thus suggesting a link with the projected dynamics used in [47] for constant metric $P$.
1) Consider a plant $\mathcal{P}$ in (3) such that SA1 holds for some compact set $\mathcal{X}$.

2) Consider an observer $\mathcal{O}$ in (4) such that Assumption 2 is satisfied for some known $t \mapsto P(t)$.

3) Choose a map $c$ such that $\mathcal{X} \subseteq \mathcal{C}_{0}$ along the lines of Lemma 1.

4) Design the function $M$ as in (14) and select $\gamma_{c}>0$ sufficiently large.

5) Add the term $M$ to the right-hand side of (4) as in (13).

6) Run the new observer (10) with $F_{m}$ defined as in (13), and initial conditions $\hat{x}(0) \in \mathcal{X}$.

7) Problem 1 is solved with $\widehat{\mathcal{X}} \supset \mathcal{C}_{\varrho}$ for some $\varrho>0$.

\section{TABLE I}

REDESIGN PROCEDURE FOR CONTINUOUS-TIME OBSERVERS

To conclude, the CT redesign summarizes in the steps given in Table I, for which we only need the knowledge of the function $c$ (which may be a degree of freedom for design, as shown later in Section VI), and the (possibly time-varying) matrix $P$ given by Assumption 2 .

\section{The Discrete-Time CASE}

\section{A. Problem Statement and Sufficient Conditions}

In this section, plant $\mathcal{P}$ is modelled as a discrete-time system of the form

$$
\mathcal{P}:\left\{\begin{aligned}
x(k+1) & =g(k, x(k), u(k), d(k)), \\
y(k) & =h(k, x(k), u(k), d(k)), \quad k \in \mathbb{N},
\end{aligned}\right.
$$

with state $x \in \mathbb{R}^{n_{x}}$, known input $u \in \mathbb{R}^{n_{u}}$, output $y \in \mathbb{R}^{n_{y}}$, and where $d \in \mathbb{R}^{n_{d}}$ is an unknown perturbation acting on the dynamics and/or the measurement. For the sake of convenience, we will denote with $x_{k}:=x(k)$ the state $x$ at discrete-time $k$. The same notation will be adopted for all variables and inputs. The plant (19) is therefore compactly written as

$$
\mathcal{P}:\left\{\begin{aligned}
x_{k+1} & =g\left(k, x_{k}, u_{k}, d_{k}\right) \\
y_{k} & =h\left(k, x_{k}, u_{k}, d_{k}\right)
\end{aligned}\right.
$$

and the observer is given by

$$
\mathcal{O}: \quad \hat{x}_{k+1}=G\left(k, \hat{x}_{k}, u_{k}, y_{k}\right)
$$

with $G: \mathbb{R}_{\geq 0} \times \mathbb{R}^{n_{x}} \times \mathcal{U} \times \mathbb{R}^{n_{y}} \rightarrow \mathbb{R}^{n_{x}}$. Similarly to the continuous-time case in Section III, the performances of observer (21) are characterized by the next Lyapunov-like conditions.

Assumption 3 There exist a (possibly time-varying) known matrix $P_{k}=P_{k}^{\top}>0$ for all $k \in \mathbb{N}$, real numbers $\bar{\lambda}>\underline{\lambda}>0$ and a continuous function $\beta_{d}: \mathbb{N} \times \mathbb{R}^{n_{x}} \times \mathbb{R}^{n_{x}} \times \mathbb{R}^{n_{u}} \times \mathbb{R}^{n_{d}} \rightarrow$ $\mathbb{R}_{\geq 0}$, such that, by defining

$$
V_{k}(x, \hat{x}):=(\hat{x}-x)^{\top} P_{k}(\hat{x}-x),
$$

the following inequalities hold for all $(k, x, \hat{x}, u, d) \in \mathbb{N} \times \mathcal{X} \times$ $\mathbb{R}^{n_{x}} \times \mathcal{U} \times \mathcal{D}$

$$
\begin{gathered}
\underline{\lambda}|\hat{x}-x|^{2} \leq V_{k}(x, \hat{x}) \leq \bar{\lambda}|\hat{x}-x|^{2}, \\
V_{k+1}\left(x_{k+1}, \hat{x}_{k+1}\right) \leq \beta_{d}(k, x, \hat{x}, u, d)
\end{gathered}
$$

with the compact notation $x_{k+1}:=g(k, x, u, d), \hat{x}_{k+1}:=$ $G(k, \hat{x}, u, h(k, x, u, d))$. 
Like Assumption 2, Assumption 3 characterizes the behavior of the estimation error $x-\hat{x}$ and similar considerations on the ISS properties can be derived, see, e.g., [53, Corollary 1]. Moreover, the function $\beta_{d}$ and the parameters $\underline{\lambda}, \bar{\lambda}$ are likewise used only for analysis purposes and not for design. In the case of a constant matrix $P, \bar{\lambda}$ and $\underline{\lambda}$ in (23) correspond to the maximum and minimum eigenvalue of $P$, respectively.

Assumption 3 is verified for the observers proposed in [4], [15]-[19],where $P$ is constant; and Kalman-like observers, see [1], [20], where $P$ is a time-varying solution to a Riccati or Lyapunov difference equation that is independent from the estimate $\hat{x}$ and uniformly upper and lower bounded in time $k$. In this case the matrix $P$ is computed online.

Similarly to the continuous-time case, we aim at solving the next problem.

Problem 2 Design a (possibly set-valued) map $G_{m}: \mathbb{N} \times$ $\mathbb{R}^{n_{x}} \times \mathbb{R}^{n_{u}} \times \mathbb{R}^{n_{y}} \rightrightarrows \mathbb{R}^{n_{x}}$ such that the following holds.

1) For any initial condition $\left(x_{0}, \hat{x}_{0}\right) \in \mathcal{X}_{0} \times \mathcal{X}$, input in $\mathcal{U}$ and perturbation in $\mathcal{D}$, the corresponding trajectory $\hat{x}_{k}$ of the modified observer

$$
\mathcal{O}_{m}: \quad \hat{x}_{k+1} \in G_{m}\left(k, \hat{x}_{k}, u_{k}, y_{k}\right)
$$

remains inside $\widehat{\mathcal{X}}$ for all $k \in \mathbb{N}$.

2) The Lyapunov function (22) satisfies

$$
V_{k+1}\left(x_{k+1}, \hat{x}_{k+1}\right) \leq \beta_{d}(k, x, \hat{x}, u, d)
$$

for any $(k, x, \hat{x}, u, d) \in \mathbb{N} \times \mathcal{X} \times \widehat{\mathcal{X}} \times \mathcal{U} \times \mathcal{D}, x_{k+1}=$ $g(k, x, u, d)$, and $\hat{x}_{k+1} \in G_{m}(k, \hat{x}, u, h(k, x, u, d))$.

Differently from the continuous-time case in Section III, we do not modify the dynamics of (21) by adding a term to the map $G$. Instead, we build $G_{m}$ by composition of the map $G$ given in (21) with a (possibly multi-valued) time-varying map $\psi_{k+1}: \mathbb{R}^{n_{x}} \rightrightarrows \mathbb{R}^{n_{x}}$, namely

$$
G_{m}(k, \cdot):=\psi_{k+1} \circ G(k, \cdot) .
$$

Remark 2 Since the mapping $\psi_{k+1}$ is applied after the mapping $G(k, \cdot)$, in the following we will assume that $\psi_{k+1}$ may depend on $P_{k+1}$. This assumption is consistent with the cases in which $P$ is obtained as solution of a Riccati difference equation for instance. It is also the reason why we index $\psi_{k+1}$ (and all the variables involved in its definition) with $k+1$ instead of $k$.

The following lemma gives a sufficient condition on the maps $\psi_{k+1}$, which allows solving Problem 2.

Lemma 3 Suppose Assumption 3 hold and that for all $k \in \mathbb{N}$, there exist a set-valued map $\psi_{k+1}: \mathbb{R}^{n_{x}} \rightrightarrows \mathbb{R}^{n_{x}}$ and a set $\mathcal{S}_{k+1} \subset \mathbb{R}^{n_{x}}$ such that

$$
\begin{gathered}
V_{k+1}\left(x, \psi_{k+1}(\hat{x})\right) \leq V_{k+1}(x, \hat{x}) \quad \forall(x, \hat{x}) \in \mathcal{X} \times \mathcal{S}_{k+1} \\
\psi_{k+1}\left(\mathcal{S}_{k+1}\right) \subseteq \widehat{\mathcal{X}}
\end{gathered}
$$

and for any $(k, x, \hat{x}, u, d) \in \mathbb{N} \times \mathcal{X} \times \widehat{\mathcal{X}} \times \mathcal{U} \times \mathcal{D}$,

$$
G(k, \hat{x}, u, h(k, x, u, d)) \in \mathcal{S}_{k+1} .
$$

Then, Problem 2 is solved with $G_{m}$ defined in (27).

Proof. From (28b), (28c), and the definition of $G_{m}$ in (27), we derive that $G_{m}(k, \hat{x}, u, h(k, x, u, d)) \subseteq \widehat{\mathcal{X}}$ for any $(k, x, \hat{x}, u, d) \in \mathbb{N} \times \mathcal{X} \times \widehat{\mathcal{X}} \times \mathcal{U} \times \mathcal{D}$. With this, item 1) of Problem 2 is satisfied. To derive the item 2) of Problem 2, we invoke (24), (28a) and the definition of $G_{m}$ in (27).

Set $\mathcal{S}_{k+1}$ contains the estimate $\hat{x}$ after applying the nominal observer (21) at iteration $k$. Therefore, it is sufficient to satisfy the redesign constraints (28a)-(28b) for $\hat{x} \in \mathcal{S}_{k+1}$.

The goal is now to find maps $\psi_{k+1}$ that verify condition (28), namely the fact that applying $\psi_{k+1}$ to $G(k, \cdot)$ does not make the Lyapunov function $V_{k+1}$ increase and that its image is contained in $\widehat{\mathcal{X}}$. The first idea we investigate is to project $G$ in $\widehat{\mathcal{X}}$, as presented in Section IV-B.

\section{B. About the Existence of a Single-Step Solution}

The next lemma shows that the function $\psi_{k+1}$ in Lemma 3 can be defined as a projection according to the norm induced by $P_{k+1}$.

Lemma 4 For any $k$ in $\mathbb{N}$, the map $\psi_{k+1}: \mathbb{R}^{n} \rightarrow \mathbb{R}^{n}$ defined by

$$
\psi_{k+1}(\hat{x}):=\underset{x \in \mathcal{C}_{0}}{\operatorname{argmin}}(\hat{x}-x)^{\top} P_{k+1}(\hat{x}-x)
$$

with $\mathcal{C}_{0}$ given in Lemma 1, is well-defined, single-valued and verifies condition (28) with $\mathcal{S}_{k+1}=\mathbb{R}^{n_{x}}$.

Proof. Take $k \in \mathbb{N}$ and define the function $\bar{c}_{k+1}: \mathbb{R}^{n_{x}} \rightarrow \mathbb{R}_{\geq 0}^{n_{c}}$ as $z \mapsto \bar{c}_{k+1}(z):=c\left(P_{k+1}^{-\frac{1}{2}} z\right)$, with $c$ given in Lemma 1 , and let $\overline{\mathcal{C}}_{0, k+1}:=\left\{z \in \mathbb{R}^{n}: \bar{c}_{k+1}(z)=0\right\}$. By linearity of the function $z \mapsto P_{k+1}^{-\frac{1}{2}} z$, and by convexity of $c_{i}$ in Lemma 1, each $i$-th component $\bar{c}_{i, k+1}$ of $\bar{c}_{k+1}$ is convex and $C^{1}$. Therefore, $\overline{\mathcal{C}}_{0, k+1}$ is convex and closed, so that the projection map $\bar{\psi}_{k+1}$ defined on $\mathbb{R}^{n}$ by

$$
\hat{z} \mapsto \bar{\psi}_{k+1}(\hat{z}):=\underset{z \in \overline{\mathcal{C}}_{0, k+1}}{\operatorname{argmin}}(\hat{z}-z)^{\top}(\hat{z}-z)
$$

takes finite values, is single-valued and verifies

$$
\left(\bar{\psi}_{k+1}(\hat{z})-z\right)^{\top}\left(\bar{\psi}_{k+1}(\hat{z})-z\right) \leq(\hat{z}-z)^{\top}(\hat{z}-z)
$$

for all $z \in \overline{\mathcal{C}}_{0, k+1}$. Since $\psi_{k+1}(\hat{x})=P_{k+1}^{-\frac{1}{2}} \bar{\psi}_{k+1}\left(P_{k+1}^{\frac{1}{2}} \hat{x}\right)$ for all $\hat{x}$ in $\mathbb{R}^{n}$ and $\mathcal{X} \subseteq \mathcal{C}_{0}$, (28a) follows with $\mathcal{S}_{k+1}=\mathbb{R}^{n}$. Equation (28b) also holds since $\mathcal{C}_{0} \subset \widehat{\mathcal{X}}$. Finally, (28c) holds because $\mathcal{S}_{k+1}=\mathbb{R}^{n}$.

With Lemmas 1, and 3, Lemma 4 implies that Problem 2 can be solved by modifying the observer along (27) with $\psi_{k+1}$ defined in (29). In other words, the estimate is brought back into $\mathcal{C}_{0}$ at each iteration through a projection of the output of the observer jump map $G$ according to the norm determined by $P_{k+1}$, thus leading to the procedure highlighted in Table II. This projection requires to solve a quadratic minimization problem with convex constraints at each step, which we now briefly discuss. 
1) Consider a plant $\mathcal{P}$ in (3) such that SA1 holds for some compact set $\mathcal{X}$.

2) Consider an observer $\mathcal{O}$ in (21) such that Assumption 3 is satisfied for some known $k \mapsto P_{k}$.

3) Choose a map $c$ such that $\mathcal{X} \subseteq \mathcal{C}_{0}$ along the lines of Lemma 1.

4) Run the new observer (25) with $G_{m}$ defined as in (27) and $\psi_{k+1}$ the projection map defined in (29), and with an initial condition $\hat{x}(0) \in \mathcal{X}$.

5) Problem 2 is solved with $\widehat{\mathcal{X}} \supset \mathcal{C}_{0}$

TABLE II

SINGLE-STEP REDESIGN PROCEDURE FOR DISCRETE-TIME OBSERVERS

Equality Constraints: If the constraint map $c$ is linear, namely $c(x)=A x-b$ with $A \in \mathbb{R}^{n_{c} \times n_{c}}$ full-rank, $\psi_{k+1}$ is explicitly defined for all $k \in \mathbb{N}$ on $\mathbb{R}^{n_{x}}$ by

$$
\psi_{k+1}(\hat{x})=\hat{x}-P_{k+1}^{-1} A^{\top}\left(A P_{k+1}^{-1} A^{\top}\right)^{-1}(A \hat{x}-b)
$$

which is an oblique projection on the constraints subspace $\left\{x \in \mathbb{R}^{n_{x}}: A x=b\right\}$ as proposed in [44] and also used in the case of time-varying linear constraints in [52]. The expression of $\psi_{k+1}$ is obtained by solving (29) using Lagrangian multipliers, which in general gives

$$
-2(\hat{x}-x)^{\top} P_{k+1}+\Lambda \frac{d c}{d x}(x)=0, \quad c(x)=0,
$$

with $\Lambda \in \mathbb{R}^{1 \times n_{c}}$. Unfortunately, those cannot be solved explicitly unless $c$ is linear. Sometimes, it can be solved numerically for instance through an iterative root search algorithm, as proposed in [50].

Inequality Constraints: In the case where the constraints can be written as convex inequalities, namely $c(x)=0 \Leftrightarrow$ $\zeta_{i}(x) \leq 0$ for $i=1 \ldots n_{c}$ with some convex maps $\zeta_{i}$ : $\mathbb{R}^{n} \rightarrow \mathbb{R}$ as a polytope (62) or an ellipse (63), then solving (29) is equivalent to solve the Karush-Kuhn-Tucker (KKT) conditions, namely finding scalars $\lambda_{1}, \ldots, \lambda_{n_{c}}$ and $x_{p} \in \mathcal{C}_{0}$ such that

$$
\begin{aligned}
& \text { 1) }-2\left(\hat{x}-x_{p}\right)^{\top} P_{k+1}+\sum_{i=1}^{n_{c}} \lambda_{i} \frac{d \zeta_{i}}{d x}\left(x_{p}\right)=0 \text {, } \\
& \text { 2) } \lambda_{i} \geq 0 \text { for all } i=1 \ldots n_{c}, \\
& \text { 3) } \lambda_{1} \zeta_{1}\left(x_{p}\right)=\ldots=\lambda_{n_{c}} \zeta_{n_{c}}\left(x_{p}\right)=0 .
\end{aligned}
$$

Various iterative algorithms such as interior-point methods, sub-gradient methods or semi-definite programming, are available to solve this convex optimization problem. In the particular case where $\mathcal{C}_{0}$ is a polytope (62) with $\zeta_{i}(x)=a_{i}^{\top} x-b_{i}$, the first condition can be solved explicitly in $x_{p}$ as a function of $\hat{x}$ and of the adjoint variables $\lambda_{i}$. Injecting its expression in the second and third conditions leads to a polyhedral partition of the state space, with a particular feasible value of $\left(\lambda_{1}, \ldots, \lambda_{n_{c}}\right)$ in each of the regions. It follows that $\psi_{k+1}$ is in that case a piecewise-linear map of the form

$$
\psi_{k+1}(\hat{x})=\hat{x}-\frac{1}{2} P_{k+1}^{-1} \sum_{i=1}^{n_{c}} \lambda_{i}(\hat{x}) a_{i}
$$

where $\lambda_{i}(\hat{x})$ depends on the zone of the partition where $\hat{x}$ is. It is interesting to note that the partition depends on the constraints $a_{i}$ and on $P_{k+1}$ only. In particular, if $P$ is constant, the partition can be determined offline beforehand, so that the computational burden at each iteration reduces to determining the region in which $\hat{x}$ lies. More details about the way this can be done efficiently can be found in [57], [58].

Towards a Multi-Step Algorithm: According to the previous analysis, we conclude that, unless $c$ is linear, an explicit solution to the minimization problem (29), namely a close form of $\psi_{k+1}$, is not available and algorithms have to be used to evaluate it. Therefore in practice, $\psi_{k+1}$ is computed as

$$
\psi_{k+1}=\psi_{k+1, \theta} \circ \cdots \circ \psi_{k+1,1},
$$

for some $\theta>1$, where each composition represents a numerical step. This implicitly requires that between any "discrete" times, we are allowed to carry out an algorithm involving several computations. As explained in [53], in the context of sampled-data systems, this means that the digital controller is fast enough with respect to the sampling frequency. Although such algorithms have been extensively studied and optimized, it may not be possible to go through all the computations in between successive sampling times when the computational power is limited or when the sampling period is small. In this case, if the algorithm is stopped prematurely, condition (28a) is not guaranteed to hold and the observer performance (26) may be destroyed. In the following section, we overcome these potential issues by proposing a map $\psi_{k+1}$ defined as (30) with $\theta>1$, but where the decrease of $V_{k+1}$ is guaranteed after each composition. This extends the idea first proposed in [53] and constitutes the main result of Section IV.

\section{Multi-Step Solution}

We introduce the map $M_{k+1}: \mathbb{R}^{n_{x}} \rightarrow \mathbb{R}^{n_{x}}$ defined by ${ }^{1}$

$$
M_{k+1}(\hat{x}):=-\gamma_{d} P_{k+1}^{-1} \frac{d c}{d x}(\hat{x})^{\top} c(\hat{x}),
$$

with $P_{k}$ coming from Assumption 3, $c$ given by Lemma 1 and $\gamma_{d}$ a strictly positive scalar to be chosen small enough, this time, contrary to Theorem 1 in Section III. We have the following result.

Lemma 5 Suppose Assumption 3 holds. For any compact set $\widehat{\mathcal{X}}_{\text {max }} \subset \mathbb{R}^{n_{x}}$, there exists $\gamma_{d}^{\star} \in \mathbb{R}_{>0} \cup\{+\infty\}$ such that, for any $0<\gamma_{d}<\gamma_{d}^{\star}$, the functions $V_{k}$ and $M_{k}$ defined in (22) and (31) satisfy

$$
\begin{aligned}
& V_{k}\left(x, \hat{x}+M_{k}(\hat{x})\right) \leq V_{k}(x, \hat{x}) \quad \forall(x, \hat{x}) \in \mathcal{X} \times \widehat{\mathcal{X}}_{\max } \\
& V_{k}\left(x, \hat{x}+M_{k}(\hat{x})\right) \leq V_{k}(x, \hat{x})-\gamma_{d} \varrho^{2} \\
& \forall(x, \hat{x}) \in \mathcal{X} \times\left(\widehat{\mathcal{X}}_{\max } \backslash \operatorname{int}\left(\mathcal{C}_{\varrho}\right)\right)
\end{aligned}
$$

for all $k \in \mathbb{N}$, and with @ given by Lemma 1 .

Proof. Given the compact set $\widehat{\mathcal{X}}_{\text {max }}$, define

$$
\begin{aligned}
\delta_{\max } & :=\max _{x \in \widehat{\mathcal{X}}_{\max }\left|\frac{d c}{d x}(x)\right|} \\
\gamma_{d}^{\star} & := \begin{cases}\frac{\lambda}{\delta_{\max }^{2}} & , \text { if } \delta_{\max } \neq 0 \\
+\infty & , \text { otherwise }\end{cases}
\end{aligned}
$$

${ }^{1}$ Observe that $M_{k}(x)$ corresponds to $M(t, x)$ defined in (14) with $P_{k}$ instead of $P(t)$. 
with $c$ given by Lemma 1 and $\underline{\lambda}$ given by Assumption 3. Note that $\delta_{\max }<\infty$ because $c$ is $\bar{C}^{1}$ and $\widehat{\mathcal{X}}_{\max }$ is compact. Take $x$ in $\mathcal{X}$ and $\hat{x}$ in $\widehat{\mathcal{X}}_{\text {max }}$. In light of (31),

$$
\begin{aligned}
& V_{k}\left(x, \hat{x}+M_{k}(\hat{x})\right) \\
& =V_{k}(x, \hat{x})+2 M_{k}(\hat{x})^{\top} P_{k}(\hat{x}-x)+M_{k}(\hat{x})^{\top} P_{k} M_{k}(\hat{x}) \\
& \leq V_{k}(x, \hat{x})-2 \gamma_{d} c(\hat{x})^{\top} \frac{d c}{d x}(\hat{x})(\hat{x}-x) \\
& \quad+\gamma_{d}^{2} c(\hat{x})^{\top} \frac{d c}{d x}(\hat{x}) P_{k}^{-1} \frac{d c}{d x}(\hat{x})^{\top} c(\hat{x}) .
\end{aligned}
$$

Since $c(x)=0$ for all $x \in \mathcal{X}$ by Lemma 1, using (2) from Lemma 2, (31), and (33), we obtain

$$
\begin{aligned}
& V_{k}\left(x, \hat{x}+M_{k}(\hat{x})\right) \\
& \quad \leq V_{k}(x, \hat{x})-\gamma_{d} c(\hat{x})^{\top}\left(2 I-\gamma_{d} \frac{d c}{d x}(\hat{x}) P_{k}^{-1} \frac{d c}{d x}(\hat{x})^{\top}\right) c(\hat{x}) \\
& \quad \leq V_{k}(x, \hat{x})-\gamma_{d}|c(\hat{x})|^{2}\left(2-\gamma_{d} \lambda^{-1} \delta_{\max }^{2}\right) \\
& \quad \leq V_{k}(x, \hat{x})-\gamma_{d}|c(\hat{x})|^{2} \leq V_{k}(x, \hat{x})
\end{aligned}
$$

in which we used $\left|P^{-1}\right| \leq \underline{\lambda}^{-1}$ and the fact that $\gamma_{d} \underline{\lambda}^{-1} \delta_{\max }^{2}<$ 1 for any $\gamma_{d}<\gamma_{d}^{\star}$. Hence (32a) holds. Moreover, when $\hat{x} \in$ $\mathbb{R}^{n_{x}} \backslash \operatorname{int}\left(\mathcal{C}_{\varrho}\right)$, we have $|c(\hat{x})| \geq \varrho$ and hence (32b) holds.

According to Lemma 5, the interest of the map $M_{k+1}$ in (31) is that, if $\widehat{\mathcal{X}}_{\text {max }}$ denotes a compact set where the estimate $\hat{x}$ remains, modifying $\hat{x}$ as $\hat{x}+M_{k+1}(\hat{x})$ makes $V_{k+1}(x, \hat{x})$ decrease no matter where the plant state $x$ actually is in $\mathcal{X}$, provided that $\gamma_{d}$ is sufficiently small (see (32a)). Furthermore, this decrease is strict when $\hat{x}$ is outside $\operatorname{int}\left(\mathcal{C}_{\varrho}\right)$, with $\mathcal{C}_{\varrho}$ defined in (1), see (32b). Since $\mathcal{C}_{\varrho} \subset \widehat{\mathcal{X}}$, our strategy is therefore to repetitively use $M_{k+1}$ to bring the estimate back into $\widehat{\mathcal{X}}$ at each iteration $k$. The redesign thus consists in correcting the state estimate $G\left(k, \hat{x}_{k}, u_{k}, y_{k}\right)$ with the map $M_{k+1}$ as long as the estimate is outside $\mathcal{C}_{\varrho}$, namely the function $\psi_{k+1, i}$ in (30) are selected as

$$
\psi_{k+1, i}(\hat{x})= \begin{cases}\hat{x}+M_{k+1}(\hat{x}) & \text { if } \hat{x} \in \mathbb{R}^{n_{x}} \backslash \mathcal{C}_{\varrho} \\ \hat{x} & \text { otherwise }\end{cases}
$$

for all $i=1 \ldots \theta$ and all $k \in \mathbb{N}$. As it can be noted by the expression of $M_{k+1}$, the correction (35) uses the gradient of the convex map $c$, namely $-d c / d x$, to bring $\hat{x}$ back to $\widehat{\mathcal{X}}$ along level sets of $V_{k+1}$. The recursive algorithm (30) stops when we cross $\mathcal{C}_{\varrho}$. This strategy is depicted in Figure 2. Note that this could not be achieved in one iteration because $\gamma_{d}$ needs to be sufficiently small to ensure that $V_{k+1}$ decreases, as shown in Lemma 5. This justifies the $\theta$ steps.

The definition of $\psi_{k+1, i}$ in (35) is not outer-semicontinuous on the boundary of $\mathcal{C}_{\varrho}$. Although it makes sense to implement (35) in practice, namely to stop the computations as soon as the estimate reaches $\mathcal{C}_{\varrho}$, we consider here a more general algorithm where the choice is left on the boundary of $\mathcal{C}_{\varrho}$ to either stop the computations or to apply $M_{k+1}$ again. This will enable us to endow our results with some nominal robustness with respect to numerical errors. The counterpart is that the map $\psi_{k+1}$ is then set-valued. A way to write this more general algorithm is to use a local state $q \in\{0,1\}$ and define on

$$
\begin{aligned}
& \mathbb{R}^{n_{x}} \times\{0,1\} \text { the set valued map }
\end{aligned}
$$

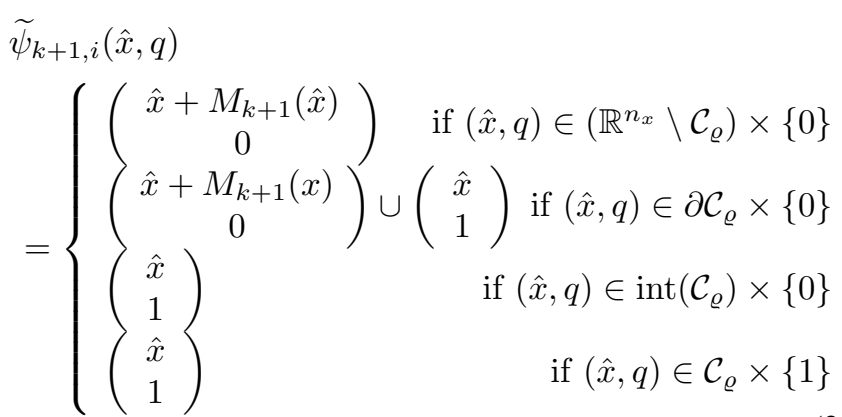

where $M_{k+1}$ is defined in (31), and $\partial \mathcal{C}_{\varrho}$ in (15). By initializing $q$ to 0 and iteratively applying $\widetilde{\psi}_{k+1, i}$, we see that as long as the $\hat{x}$ is outside $\mathcal{C}_{\varrho}$, it is corrected using the map $M_{k+1}$ and $q$ is kept equal to 0 . If $\hat{x}$ reaches the boundary of $\mathcal{C}_{\varrho}$, there is a choice between correcting it with $M_{k+1}$ or keeping it the same. In that latter case or if the estimate reaches the interior of $\mathcal{C}_{\varrho}, q$ is switched to 1 and $\hat{x}$ is kept constant until the end of the $\theta$ jumps. In other words, $\widetilde{\psi}_{k+1, i}$ becomes the identity and the latest estimate is kept. The following result shows that by applying this algorithm the component $\hat{x}$ ends up in $\widehat{\mathcal{X}}$ while making $V_{k+1}$ decrease. To this end, we define, for $k$ in $\mathbb{N}$, the maps $\widetilde{\psi}_{k+1}$ as

$$
\widetilde{\psi}_{k+1}=\widetilde{\psi}_{k+1, \theta} \circ \cdots \circ \widetilde{\psi}_{k+1,1},
$$

with $\widetilde{\psi}_{k+1, i}$ defined in (36), and, for $k$ in $\mathbb{N}$, the map $\psi_{k+1}$ : $\mathbb{R}^{n_{x}} \rightarrow \mathbb{R}^{n_{x}}$ as

$$
\psi_{k+1}(\hat{x})=\operatorname{Proj}_{\hat{x}} \widetilde{\psi}_{k+1}(\hat{x}, 0)
$$

with $\widetilde{\psi}_{k+1}$ defined in (37). The map $\operatorname{Proj}_{\hat{x}}$ so defined is simply the selection of the $n_{x}$ first components of $\widetilde{\psi}_{k+1}(\hat{x}, 0)$ of dimension $n_{x}+1$, namely the removal of the inner state $q$.

Finally, in order to state the main result of this section, let us define the real number $v_{\max }$ as

$$
v_{\max }:=\sup _{(k, x, \hat{x}, u, d) \in \mathbb{N} \times \mathcal{X} \times \hat{\mathcal{X}} \times \mathcal{U} \times \mathcal{D}} \beta_{d}(k, x, \hat{x}, u, d),
$$

with $\beta_{d}$ given by Assumption 3, and according to its definition, for any $k \in \mathbb{N}$, let us define the sets $\widehat{\mathcal{X}}_{k+1, \max }$

$$
\widehat{\mathcal{X}}_{k+1, \max }:=\left\{\hat{x} \in \mathbb{R}^{n_{x}}: \exists x \in \mathcal{X}, V_{k+1}(x, \hat{x}) \leq v_{\max }\right\} .
$$

Theorem 2 Suppose Assumption 3 holds and that $v_{\max }$ defined in (39) is finite. Then, there exists $\gamma_{d}^{\star} \in \mathbb{R}_{>0} \cup\{+\infty\}$ such that, for any $\gamma_{d}$ satisfying $0<\gamma_{d}<\gamma_{d}^{\star}$, there exists a nonnegative scalar $\theta$ such that for all $k \in \mathbb{N}$, the map $\psi_{k+1}$ defined in (38) is outer semi-continuous and verifies conditions (28) of Lemma 3 with $\mathcal{S}_{k+1}=\widehat{\mathcal{X}}_{k+1, \max }$ defined in (40), namely Problem 2 is solved.

Proof. The proof is postponed to Appendix A.

In order to use $\psi_{k+1}$ defined in (38), we need to be able to make (at most) $\theta+1$ recursive computations at each discrete step $k$. Note, however, that in this case, the Lyapunov function decreases even if we perform less than $\theta+1$ steps. Therefore, in case of limited computational power, it is still interesting 


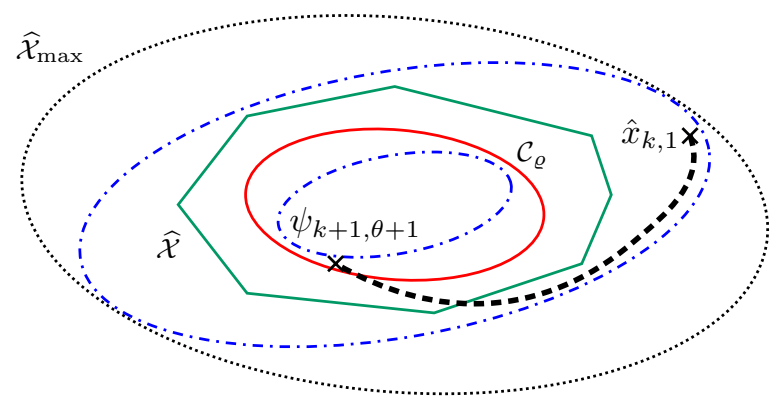

Fig. 2. Refining strategy (30) with (35), starting from $\hat{x}_{k, 1}=$ $G\left(k, \hat{x}_{k}, u_{k}, y_{k}\right)$. Dash-dotted blue ellipses: Lyapunov level sets of $V_{k+1}$. Red ellipsoid: $\mathcal{C}_{\varrho}$. Green polygon: $\widehat{\mathcal{X}}$. Dotted black ellipse: $\widehat{\mathcal{X}}_{\text {max }}$, a compact set where the solution is proved to remain. Dashed black line: path of $\hat{x}_{k, i+1}=\psi_{k, i}\left(\hat{x}_{k, i}\right), i=1, \ldots, \theta$.

to do as many $\theta_{m} \in\{0, \ldots, \theta\}$ steps as possible, as long as the estimate is outside of $\mathcal{C}_{\varrho}$ : the Lyapunov function will decrease by $\theta_{m} \gamma_{d} \varrho^{2}$ which will make the algorithm converge faster and make the estimate stay closer to $\widehat{\mathcal{X}}$. This is a clear advantage compared to the projection-based algorithm presented in Section IV-B.

As detailed in the proof of Theorem 2, the number $\theta$ defined in (70) gives the largest number of refining steps needed to bring $\hat{x}$ in $\mathcal{C}_{\varrho}$ and increases with the size of the sets $\widehat{\mathcal{X}}_{k+1 \text {,max }}$. In practice, this number may be significantly smaller, as the analysis is made on the conservative assumption that at each step, the decrease rate of $V_{k+1}$ is $\gamma_{d} \varrho^{2}$. Note that an estimate of $\gamma_{d}^{\star}$ is given in (33). According to Lemma 5, the true decrease is $\gamma_{d}|c(\hat{x})|^{2}$, which is larger than $\gamma_{d} \varrho^{2}$ when $\hat{x}$ is far from $\mathcal{C}_{\varrho}$, due to the convexity assumption in Lemma 1 . In fact, the definition of $\widetilde{\psi}_{k+1, i}$ in (36) provides, from a computational point of view, a fast way to interrupt the algorithm once $\hat{x}$ is in $\mathcal{C}_{\varrho}$.

An important observation is that although $\hat{x}_{k+1}$ is in $\widehat{\mathcal{X}}$, the intermediary estimates $\hat{x}_{k, i}$, obtained while implementing (37), can leave this set. However, according to the proof of Theorem 2 , they remain in a compact set $\widehat{\mathcal{X}}_{\max }$ properly defined. Therefore, $\widehat{\mathcal{X}}_{\max }$ gives the magnitude of the numbers to be computed throughout the algorithm. This information can be useful to optimize the variables encoding and increase numerical precision in case of limited memory, in embedded systems for instance. Actually, this set could be made more precise by considering the evolution of $V_{k}$ given by (24) and the maximal initial error $e_{0}=\hat{x}_{0}-x_{0}$.

To conclude, this multi-step DT redesign may be summed up as in Table III. Note, that, as in the CT-case, only the knowledge of $P_{k}$ and $c$ are required.

Remark 3 We might wonder whether $\psi_{k+1}$ defined in Theorem 2 is an iterative way of approaching $\psi_{k+1}$ as defined in Lemma 4 when the number of iterations, namely $\theta$, goes to infinity. It turns out on examples that it is not. Actually, by compactness of $\mathcal{C}_{0}$ and convexity of $c$, it is possible to show by contradiction that, $\psi_{k+1}(\hat{x})$ defined in (29) is not the only solution to (28a) on $\mathbb{R}^{n}$. More precisely, given $\hat{x}$ in $\mathbb{R}^{n}$, there exists a neighborhood of $\psi_{k+1}(\hat{x})$ such that (28a) still holds.
1) Consider a plant $\mathcal{P}$ in (3) such that SA1 holds for some compact set $\mathcal{X}$.

2) Consider an observer $\mathcal{O}$ in (21) such that Assumption 3 is satisfied for some known $k \mapsto P_{k}$

3) Choose a map $c$ such that $\mathcal{X} \subseteq \mathcal{C}_{0}$ along the lines of Lemma 1 .

4) Design the function $M_{k+1}$ as in (31), and select $\gamma_{d}>0$ small enough.

5) Design the function $\psi_{k+1}$ as in (30) with $\psi_{k+1, i}$ defined in (35); alternatively, if outer semi-continuity is sought, select $\psi_{k+1}$ as in (38), with $\widetilde{\psi}_{k+1}$ defined in (37) and $\widetilde{\psi}_{k+1, i}$ in (36).

6) Select $\theta$ large enough and run the new observer (25) with $G_{m}$ defined as in (27) and $\psi_{k+1}$ as in the previous step, and with an initial condition $\hat{x}(0) \in \mathcal{X}$.

7) Problem 2 is solved with $\widehat{\mathcal{X}} \supset \mathcal{C}_{\varrho}$.

TABLE III

Multi-steP REDESIGN PROCEDURE FOR DisCRETE-TIME OBSERVERS

Remark 4 The robustness of the modified observer with respect to the perturbation $d$ is characterized by $\beta_{d}$ in (26), which is the same as for the initial observer. In fact, the added computational steps due to $\psi_{k+1}$ are not affected by perturbations, they are carried out independently from the plant and the observer: their only role is to bring the estimate back into the viable set $\widehat{\mathcal{X}}$. Therefore, the only robustness we could be concerned about for conditions (28), is with respect to numerical errors in the recursive computation of $\psi_{k+1}$. In light of the outer semi-continuity of $\widetilde{\psi}_{k+1, i}$, the maps $\widetilde{\psi}_{k+1}$, $\psi_{k+1}$ (and thus $F_{m}$ ) are outer semi-continuous and locally bounded, which ensure sequential compactness of solutions to (25), and thus robustness of the result of Theorem 2 with respect to numerical errors, see [22, Assumption 6.5].

\section{THE HYBRID CASE}

\section{A. Problem Statement}

In this section, we exploit the results of the previous two sections to address the scenario where plant $\mathcal{P}$ is given by the hybrid model (see [22], [59])

$$
\mathcal{P}:\left\{\begin{array}{rll}
\dot{\tau} & \in f_{\tau}(\tau) \\
\dot{x} & \in f(\tau, x, u, d)
\end{array}\right\} \quad(\tau, x, u, d) \in C
$$

where $C$ and $D$ are the flow and the jump sets respectively, $\left(f_{\tau}, f\right)$ and $\left(g_{\tau}, g\right)$ are the flow and jump maps respectively, $x$ and $y$ are respectively the state and the output of the plant. Variable $\tau \in \mathbb{R}^{n_{\tau}}$ is another state variable ${ }^{2}$, which is supposed to be available for the observer design, and which can be a timer keeping track of the time and/or jumps to model the time-dependency of $\mathcal{F}$ and $\mathcal{G}$. For instance, $\tau, n_{\tau}=1$, could model the time $t$ with $f_{\tau} \equiv 1$, and $g_{\tau}=\mathrm{Id}$, or a counter of jumps with $f_{\tau} \equiv 0$ and $g_{\tau}(\tau)=\tau+1$, or the time elapsed since the previous jump with $f_{\tau} \equiv 1$ and $g_{\tau} \equiv 0$. Due to the presence of $\tau$, we need to reformulate SA1 as follows.

\footnotetext{
${ }^{2}$ Its dynamics $f_{\tau}$ and $g_{\tau}$ could also depend on $(x, u, d)$ as long as $\tau$ is available to the observer.
} 
Standing Assumption 3 (SA1') There exist subsets $\mathcal{T}_{0} \subset$ $\mathbb{R}^{n_{\tau}}, \mathcal{T} \subset \mathbb{R}^{n_{\tau}}, \mathcal{X}_{0} \subset \mathbb{R}^{n_{x}}, \mathcal{U} \subset \mathbb{R}^{n_{u}}, \mathcal{D} \subset \mathbb{R}^{n_{d}}$, and a compact subset $\mathcal{X} \subset \mathbb{R}^{n_{x}}$, such that any maximal solution ([59]) of (41) initialized in $\mathcal{T}_{0} \times \mathcal{X}_{0}$, with input in $\mathcal{U}$ and perturbation in $\mathcal{D}$, remains in $\mathcal{T} \times \mathcal{X}$ at all forward times.

Since we are interested only in solutions satisfying SA1', in the following we suppose that

$$
C \subseteq \mathcal{T} \times \mathcal{X} \times \mathcal{U} \times \mathcal{D}, \quad D \subseteq \mathcal{T} \times \mathcal{X} \times \mathcal{U} \times \mathcal{D} .
$$

Furthermore, to simplify the following statements, we denote $\mathcal{T}_{c}$ and $\mathcal{T}_{d}$ the projections of $C$ and $D$ on the first $n_{\tau}$ components, namely sets verifying $\mathcal{T}_{c} \cup \mathcal{T}_{d} \subseteq \mathcal{T}$ and

$$
\begin{aligned}
& (\tau, x, u, d) \in C \quad \Longrightarrow \quad \tau \in \mathcal{T}_{c} \\
& (\tau, x, u, d) \in D \quad \Longrightarrow \quad \tau \in \mathcal{T}_{d} .
\end{aligned}
$$

We assume now that we know an observer whose jumps are synchronized with those of the plant, i.e., such that the complete system can be written as

$$
\begin{aligned}
& \dot{\tau} \in f_{\tau}(\tau) \\
& \dot{x} \in f(\tau, x, u, d) \\
& \dot{\hat{x}} \in F(\tau, \hat{x}, u, h(\tau, x, u, d)) \\
& \tau^{+} \in g_{\tau}(\tau) \\
& x^{+} \in g(\tau, x, u, d) \\
& \hat{x}^{+} \in G(\tau, \hat{x}, u, h(\tau, x, u, d)) \\
& (\tau, x, u, d) \in C, \\
& \hat{x} \in \mathbb{R}^{n_{x}}, \\
& (\tau, x, u, d) \in D \\
& \hat{x} \in \mathbb{R}^{n_{x}} \text {, }
\end{aligned}
$$

with flow map $\mathcal{F}:=\left(f_{\tau}, f, F\right)$ and jump map $\mathcal{G}:=\left(g_{\tau}, g, G\right)$. It is important to notice that the domain of solutions to (44) are determined by $(\tau, x, u, d)$ only: for any solution $(\tau, x, \hat{x})$ of (44), $(\tau, x)$ is solution to (41) so that $\hat{x}$ inherits the domain of $(\tau, x)$, at least as long as it is defined. This implicitly assumes that the jump times of (41) are known and can be used in the observer. This context covers a lot of designs, as detailed below. As before, we assume that the observer has been designed based on a quadratic Lyapunov function and we state the following assumption.

Assumption 4 There exist a known differentiable $P(\tau)=$ $P(\tau)^{\top} \in \mathbb{R}^{n_{x} \times n_{x}}>0$ for $\tau \in \mathbb{R}^{n_{\tau}}$, positive real numbers $\bar{\lambda}>\underline{\lambda}>0$, functions $\beta_{c}: \mathbb{R}^{n_{\tau}} \times \mathbb{R}^{n_{x}} \times \mathbb{R}^{n_{x}} \times \mathbb{R}^{n_{u}} \times \mathbb{R}^{n_{d}} \rightarrow \mathbb{R}$ $\beta_{d}: \mathbb{R}^{n_{\tau}} \times \mathbb{R}^{n_{x}} \times \mathbb{R}^{n_{x}} \times \mathbb{R}^{n_{u}} \times \mathbb{R}^{n_{d}} \rightarrow \mathbb{R}_{\geq 0}$, such that the map defined on $\mathbb{R}^{n_{\tau}} \times \mathbb{R}^{n_{x}} \times \mathbb{R}^{n_{x}}$ by

$$
V(\tau, x, \hat{x}):=(\hat{x}-x)^{\top} P(\tau)(\hat{x}-x),
$$

verifies for all $(\tau, x, \hat{x}) \in \mathcal{T} \times \mathcal{X} \times \mathbb{R}^{n_{x}}$,

$$
\underline{\lambda}|\hat{x}-x|^{2} \leq V(\tau, x, \hat{x}) \leq \bar{\lambda}|\hat{x}-x|^{2},
$$

and for all $\hat{x} \in \mathbb{R}^{n_{x}}$,

$$
\begin{array}{cr}
L_{\mathcal{F}} V(\tau, x, \hat{x}, u, d) \leq \beta_{c}(\tau, x, \hat{x}, u, d) & \forall(\tau, x, u, d) \in C, \\
V(\mathcal{G}(\tau, x, \hat{x}, u, d)) \leq \beta_{d}(\tau, x, \hat{x}, u, d) & \forall(\tau, x, u, d) \in D .
\end{array}
$$

The results of this section cover the following cases.

- A continuous-time plant with a sampled-data hybrid observer with $g=\mathrm{Id}$, and $\tau$ a timer determining the sampling times: see, among others, [24] for periodic sampling with $P$ constant, [25] for aperiodic sampling with $P$ time-varying, but also [26]-[28], [60] and references therein.

- A hybrid plant with known jump times and a hybrid synchronized observer as in the context of switched systems with common $P$ [29] or switched $P$ [30], [31] and $\tau$ denoting the switching signal, impulsive systems [32], or general linear hybrid systems [33] among others.

- A continuous-time plant with a continuous-time observer as in Section III, with $\mathcal{G} \equiv \emptyset, D=\emptyset, \tau=t \in \mathbb{R}, \mathcal{F}$ defined in (8), and $C=\mathbb{R} \times \mathbb{R}^{n_{x}} \times \mathbb{R}^{n_{u}} \times \mathbb{R}^{n_{d}}$. In this context, we aim at redesigning the continuous-time observer by using the technique in Section III, thus remaining in the context of continuous-time observers, or by using the technique in Section IV, thus obtaining a hybrid observer. An example of the two different constructions is given in Section VII-A.

- A continuous-time plant with a hybrid observer, see for instance [34]-[36].

- A discrete-time plant with a discrete-time observer as in Section IV with $\mathcal{F} \equiv \emptyset, C=\emptyset, \tau=k \in \mathbb{N}, \mathcal{G}=(\tau+$ $1, f, F)$ with $f$ and $F$ defined in (20)-(21), and $D=\mathbb{N} \times$ $\mathbb{R}^{n_{x}} \times \mathbb{R}^{n_{u}} \times \mathbb{R}^{n_{d}}$.

\section{B. Nominal Redesign}

Using the tools previously developed for flow/jump redesign in Sections III and IV, we want to modify $F$ and $G$ so that the component $\hat{x}$ of the modified system

$$
\begin{aligned}
& \dot{\tau} \in f_{\tau}(\tau) \\
& \dot{x} \in f(\tau, x, u, d) \quad\}(\tau, x, u, d) \in C, \\
& \left.\dot{\hat{x}} \in F_{m}(\tau, \hat{x}, u, h(\tau, x, u, d))\right\} \quad \hat{x} \in \mathbb{R}^{n_{x}}, \\
& \tau^{+} \in g_{\tau}(\tau) \\
& x^{+} \in g(\tau, x, u, d) \\
& \left.\hat{x}^{+} \in G_{m}(\tau, \hat{x}, u, h(\tau, x, u, d))\right\} \quad \hat{x} \in \mathbb{R}^{n_{x}}
\end{aligned}
$$

with flow map $\mathcal{F}_{m}=\left(f_{\tau}, f, F_{m}\right)$ and jump map $\mathcal{G}_{m}=$ $\left(g_{\tau}, g, G_{m}\right)$, remains in $\widehat{\mathcal{X}}$ and the performances stated in Assumption 4 are preserved when $\hat{x} \in \widehat{\mathcal{X}}$. An additional constraint we must add is that the maximal solutions of the modified observer (48) should be defined at least as long as the corresponding maximal solutions of the initial observer (44). Otherwise, a trivial solution would be to take $F_{m}=F$, $G_{m}=G$ and restrict the flow/jump maps to $\hat{x} \in \widehat{\mathcal{X}}$, but the solutions could then die prematurely when $\hat{x}$ points out $\widehat{\mathcal{X}}$, thus altering the observer performances. Inspired by the previous sections, for $(\tau, x, \hat{x}, u, y) \in \mathbb{R}^{n_{\tau}} \times \mathbb{R}^{n_{x}} \times \mathbb{R}^{n_{x}} \times \mathbb{R}^{n_{u}} \times \mathbb{R}^{n_{y}}$, we define

$$
\begin{aligned}
F_{m}(\tau, \hat{x}, u, y) & :=F(\tau, \hat{x}, u, y)+M_{c}(\tau, \hat{x}) \\
G_{m}(\tau, \hat{x}, u, y) & :=\psi\left(g_{\tau}(\tau), G(\tau, \hat{x}, u, y)\right)
\end{aligned}
$$

with $M_{c} \equiv M$ defined in (14) and $\psi$ a map to be defined. In fact, this map $\psi\left(g_{\tau}(\tau), \cdot\right)$ is going to be the equivalent of $\psi_{k+1}$ used in Section IV, which is coherent with the fact that for discrete systems, $\tau$ stands for $k$ and $g_{\tau}(\tau)$ for $k+1$.

Consider

$$
F_{\max }:=\sup _{(\tau, \hat{x}, u, x, d) \in \mathcal{T}_{c} \times \partial \mathcal{C}_{\varrho} \times \mathcal{U} \times \mathcal{X} \times \mathcal{D}}|F(\tau, \hat{\mathcal{D}}, u, h(t, x, u, d))|,
$$

with $\mathcal{C}_{\varrho}$ defined in (15). Then, we have the following result. 
Lemma 6 Suppose that: Assumption 4 holds; $\delta_{\min }$ defined in (16) is strictly positive; $F_{\max }$ defined in (50) is finite. Fix $\gamma_{c}>\gamma_{c}^{\star}>0$ with $\gamma_{c}^{\star}$ defined in (18) with $F_{\max }$ given in (50) and $\bar{\lambda}$ in Assumption 4. Assume there exist maps $\psi$ : $\mathbb{R}^{n_{\tau}} \times \mathbb{R}^{n_{x}} \rightrightarrows \mathbb{R}^{n_{x}}$ and $\mathcal{S}: \mathbb{R}^{n_{\tau}} \rightrightarrows \mathbb{R}^{n_{x}}$ such that for all $\tau \in \mathcal{T}_{d}$,

$$
\begin{aligned}
& V\left(\tau^{+}, x, \psi\left(\tau^{+}, \hat{x}\right)\right) \leq V\left(\tau^{+}, x, \hat{x}\right) \\
& \forall\left(\tau^{+}, x, \hat{x}\right) \in g_{\tau}(\tau) \times \mathcal{X} \times \mathcal{S}(\tau) \\
& \psi\left(g_{\tau}(\tau), \mathcal{S}(\tau)\right) \subseteq \mathcal{C}_{\varrho}
\end{aligned}
$$

and moreover, for any $(\tau, x, u, d) \in D$ and any $\hat{x} \in \widehat{\mathcal{X}}$, we have

$$
G(\tau, \hat{x}, u, h(\tau, x, u, d)) \in \mathcal{S}(\tau) .
$$

Then, the functions $V, \mathcal{F}_{m}, \mathcal{G}_{m}$, defined by (45), (49), satisfy

$$
\begin{aligned}
& L_{\mathcal{F}_{m}} V(\tau, x, \hat{x}, u, d) \leq \beta_{c}(\tau, x, \hat{x}, u, d) \\
& \forall \hat{x} \in \widehat{\mathcal{X}}, \forall(\tau, x, u, d) \in C, \\
& V\left(\mathcal{G}_{m}(\tau, x, \hat{x}, u, d)\right) \leq \beta_{d}(\tau, x, \hat{x}, u, d) \\
& \forall \hat{x} \in \hat{\mathcal{X}}, \forall(\tau, x, u, d) \in D .
\end{aligned}
$$

In addition, any maximal solution $(\tau, x, \hat{x})$ to (44) initialized in $\mathcal{T}_{0} \times \mathcal{X}_{0} \times \mathcal{X}$ with input in $\mathcal{U}$ and perturbation in $\mathcal{D}$, is such that $\hat{x}$ remains in $\widehat{\mathcal{X}}$ at all times and $(\tau, x)$ is a maximal solution to (41).

Proof. Equation (52a) follows in the same way as in the proof of Theorem 1 using (42). Equation (52b) follows directly from (49b), (51a) and (51c). Besides, combining (49b), (51a) and (51b), we get

$$
G_{m}(\tau, \hat{x}, u, h(\tau, x, u, d)) \subseteq \mathcal{C}_{\varrho} \quad \forall(\tau, x, u, d) \in D, \hat{x} \in \widehat{\mathcal{X}} .
$$

Now take a solution $(\tau, x, \hat{x})$ to (44) initialized in $\mathcal{T}_{0} \times \mathcal{X}_{0} \times \mathcal{X}$. Because of the flow/jump sets are the same as the initial plant, $(\tau, x)$ is solution to (41) and from SA1', $(\tau, x)$ is in $\mathcal{T} \times \mathcal{X}$. Since $\mathcal{X}_{0} \subset \mathcal{X} \subset \mathcal{C}_{\varrho}, \hat{x}(0,0) \in \mathcal{C}_{\varrho}$. As long as (44) flows for $t \in\left[0, t_{1}\right], \hat{x}(t, 0)$ remains in $\mathcal{C}_{\varrho}$ by Theorem 1 . Then, at the first jump, $\hat{x}\left(t_{1}, 0\right) \in \mathcal{C}_{\varrho} \subset \widehat{\mathcal{X}}$, and by definition of solutions $(\tau, x, u, d)\left(t_{1}, 0\right) \in D$, so that, from (53), $\hat{x}\left(t_{1}, 1\right) \in \mathcal{C}_{\varrho}$. Repeating the same reasoning from that initial condition, we deduce that $\hat{x}$ remains in $\mathcal{C}_{\varrho} \subset \widehat{\mathcal{X}}$. Because the flow/jump conditions of (48) are independent from $\hat{x}$, any maximal solution $(\tau, x, \hat{x})$ of (44) is such that $(\tau, x)$ is maximal for (41) unless $\hat{x}$ explodes in finite time during flow. This latter case is impossible since $\widehat{\mathcal{X}}$ is compact.

Lemma 6 says that, if the map $\psi$ used in $G_{m}$ in (49b) verifies conditions (51), the Lyapunov properties of the observer are preserved and the estimate $\hat{x}$ remains in $\hat{\mathcal{X}}$. More importantly, the estimate is defined as long as the underlying plant solution is, which was not necessarily the case of the initial observer (44). Therefore, the modification does not alter the properties of the solutions and keeps $\hat{x}$ in $\widehat{\mathcal{X}}$.

Remark 5 Conditions (51) are trivially satisfied with $\psi(\tau, \cdot)=$ Id if $G(\tau, \hat{x}, u, h(\tau, x, u, d)) \in \mathcal{C}_{\varrho}$ for all $(\tau, x, u, d) \in D$ and $\hat{x} \in \widehat{\mathcal{X}}$. In that case, $G_{m}=G$, namely only the flow map is modified. Otherwise, it is necessary to modify the jump map in order to guarantee solutions are in $\widehat{\mathcal{X}}$ after the jumps.

Similarly to Lemma 4 , the map $\psi: \mathbb{R}^{n_{\tau}} \times \mathbb{R}^{n_{x}}$ defined by

$$
\psi(\tau, \hat{x}):=\underset{x \in \mathcal{C}_{0}}{\operatorname{argmin}}(\hat{x}-x)^{\top} P(\tau)(\hat{x}-x)
$$

satisfies conditions (51) with $\mathcal{S}(\tau)=\mathbb{R}^{n_{x}}$ for all $\tau \in \mathbb{R}^{n_{\tau}}$. However, as mentioned in Section IV-B, this map may be difficult to implement. Alternatively, we can follow the design of Section IV-C. For that, we introduce the map $M_{d}: \mathbb{R}^{n_{\tau}} \times$ $\mathbb{R}^{n_{x}} \rightarrow \mathbb{R}^{n_{x}}$ defined by

$$
M_{d}(\tau, \hat{x}):=-\gamma_{d} P(\tau)^{-1} \frac{d c}{d x}(\hat{x})^{\top} c(\hat{x}),
$$

with $\gamma_{d}$ a strictly positive scalar, and $P(\tau)$ coming from Assumption 4. As in Theorem 2, if

$$
v_{d, \text { max }}:=\max _{(\tau, x, \hat{x}, u, d) \in \mathcal{T}_{d} \times \mathcal{X} \times \widehat{\mathcal{X}} \times \mathcal{U} \times \mathcal{D}} \beta_{d}(\tau, x, \hat{x}, u, d)
$$

is finite and by choosing $\gamma_{d}$ sufficiently small, we can bring $\hat{x}^{+}=G(\tau, \hat{x}, u, y)$ back into $\mathcal{C}_{\varrho} \subset \widehat{\mathcal{X}}$ after each jump, without making $V$ increase, by repetitively using $M_{d}\left(\tau^{+}, \cdot\right)$ a finite number of times. In order to avoid heavy notations, we do not explicitly rewrite the map $\psi$ gendered by this method which is a direct transposition of Section IV-C. Instead, we notice that, in the hybrid context, such a composition of functions can be more easily modeled by a succession of jumps that can directly be encoded in the hybrid system. This leads to the hybrid redesign presented in the following section.

\section{Hybrid Redesign}

Inspired by (36), we add a toggle state $q$ to the observer state $\hat{x}$, and define the map $\widetilde{\psi}: \mathbb{R}^{n_{\tau}} \times \mathbb{R}^{n_{x}} \rightarrow \mathbb{R}^{n_{x}} \times\{0,1\}$ as

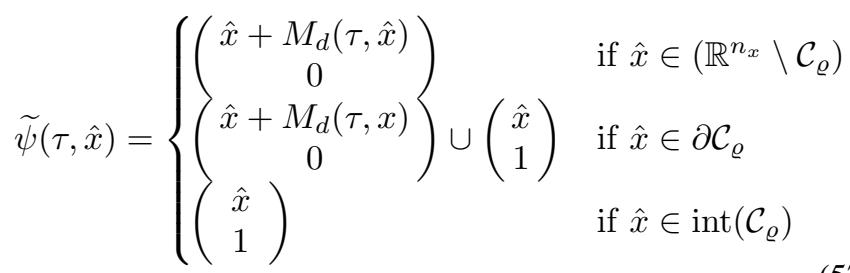

we consider the new observer dynamics

$$
\begin{aligned}
& (\dot{\tau}, \dot{x}, \dot{\hat{x}}, \dot{q})^{\top} \in \widetilde{\mathcal{F}}(\tau, x, \hat{x}, u, d), \quad(\tau, x, \hat{x}, q, u, d) \in \widetilde{C}, \\
& \left(\tau^{+}, x^{+}, \hat{x}^{+}, q^{+}\right)^{\top} \in \widetilde{\mathcal{G}}(\tau, x, \hat{x}, u, d), \quad(\tau, x, \hat{x}, q, u, d) \in \widetilde{D},
\end{aligned}
$$

with $\widetilde{\mathcal{F}}=\left(f_{\tau}, f, F, 0\right)$,

$$
\widetilde{C}:=\{(\tau, x, \hat{x}, q, u, d):(\tau, x, u, d) \in C, \hat{x} \in \widehat{\mathcal{X}}, q=1\},
$$

$\widetilde{D}$ is the domain of definition of the jump map defined by

$$
\widetilde{\mathcal{G}}= \begin{cases}\widetilde{\mathcal{G}}_{11} & \text { if } q=1,(\tau, x, u, d) \in D, \hat{x} \in \operatorname{int}(\widehat{\mathcal{X}}) \\ \widetilde{\mathcal{G}}_{11} \cup \widetilde{\mathcal{G}}_{10} & \text { if } q=1,(\tau, x, u, d) \in D, \hat{x} \in \partial \widehat{\mathcal{X}} \\ \tilde{\mathcal{G}}_{10} & \text { if } q=1,(\tau, x, u, d) \in D, \hat{x} \in \mathbb{R}^{n_{x}} \backslash \widehat{\mathcal{X}} \\ & \text { or } q=1,(\tau, x, u, d) \in \operatorname{cl}(C) \backslash D, \\ & \hat{x} \in \operatorname{cl}\left(\mathbb{R}^{n_{x}} \backslash \widehat{\mathcal{X}}\right) \\ \widetilde{\mathcal{G}}_{0} & \text { if } q=0,(\tau, x, u, d) \in \operatorname{cl}(C) \cup D, \\ & \end{cases}
$$


with

$$
\begin{aligned}
\widetilde{\mathcal{G}}_{11}(\tau, x, \hat{x}, u, d):=\left(g_{\tau}(\tau), g(\tau, x, u, d),\right. \\
G(\tau, \hat{x}, u, h(\tau, x, u, d)), 1) \\
\widetilde{\mathcal{G}}_{10}(\tau, x, \hat{x}, u, d):=(\tau, x, \hat{x}, 0) \\
\widetilde{\mathcal{G}}_{0}(\tau, x, \hat{x}, u, d):=(\tau, x, \widetilde{\psi}(\tau, \hat{x}))
\end{aligned}
$$

Still assuming that $v_{d, \max }$ defined in (56) is finite, we consider the compact sets

$$
\begin{aligned}
\widehat{\mathcal{X}}_{d, \max }:=\left\{\hat{x} \in \mathbb{R}^{n_{x}}: \exists \tau \in \mathcal{T}_{d},\right. \\
\left.\exists x \in \mathcal{X}, V(\tau, x, \hat{x}) \leq v_{d, \max }\right\} .
\end{aligned}
$$

Theorem 3 Select $\gamma_{d}>0$ satisfying $\gamma_{d}<\gamma_{d}^{\star}$ defined in (33)-(34) with $\widehat{\mathcal{X}}_{\max }:=\widehat{\mathcal{X}} \cup \widehat{\mathcal{X}}_{d, \max }$. Then, any solution $\phi=(\tau, x, \hat{x}, q)$ of (58) initialized in $\mathcal{T}_{0} \times \mathcal{X}_{0} \times \widehat{\mathcal{X}} \times\{1\}$, verifies $\operatorname{dom} \phi=\mathcal{D}_{1} \cup \mathcal{D}_{0}$, with $\mathcal{D}_{0}$ and $\mathcal{D}_{1}$ such that $q(t, j)=0$ for all $(t, j) \in \mathcal{D}_{0}$ and $q(t, j)=1$ for all $(t, j) \in \mathcal{D}_{1}$, and the following.

- The projection of $\mathcal{D}_{0}$ on $t$ is of null Lebesgue measure.

- The hybrid arc $s=(\tau, x, \hat{x}, u, d)_{\mid \mathcal{D}_{1}}$ verifies $\hat{x}(t, j) \in \widehat{\mathcal{X}}$ for all $(t, j) \in \operatorname{dom} s$, and, for all $(t, j) \in \operatorname{dom} s$,

$$
\frac{d}{d t} V((\tau, x, \hat{x})(t, j)) \leq \beta_{c}((\tau, x, \hat{x}, u, d)(t, j))
$$

and for all $(t, j) \in \operatorname{dom} s$ such that $(t, j+1) \in \operatorname{dom} s$,

$$
V((\tau, x, \hat{x})(t, j+1)) \leq \beta_{d}((\tau, x, \hat{x}, u, d)(t, j)) .
$$

- Removing the trivial jumps from $(\tau, x)$ gives a maximal solution of the plant (41).

The first item says that the part $\mathcal{D}_{0}$ of the time domain where $q=0$ consists only of jumps (no flow), and the second item, that along the solutions restricted to the rest of the time domain $\mathcal{D}_{1}$ where $q=1, \hat{x} \in \widehat{\mathcal{X}}$ and the Lyapunov properties are preserved. In other words, we are only adding jumps, "correction phases" where $q=0$, designed to bring $\hat{x}$ back into $\widehat{\mathcal{X}}$ whenever it attempts to leave $\widehat{\mathcal{X}}$. Besides, the third item says that modulo the added jumps in $\mathcal{D}_{0}$, we indeed browse the entire domain of the underlying plant solution, i.e. our modification does not make solutions stop prematurely. The following proof further explains the behavior of the solutions.

Proof. As long as $\hat{x} \in \operatorname{int}(\widehat{\mathcal{X}}), q$ remains equal to 1 , and $(\tau, x, \hat{x})$ evolves according to the initial observer dynamics (44) with $\widetilde{\mathcal{F}}=(\mathcal{F}, 0)$ and $\widetilde{\mathcal{G}}=\widetilde{\mathcal{G}}_{11}=(\mathcal{G}, 1)$. If $\hat{x}$ reaches the boundary of $\widehat{\mathcal{X}}$ after flow or jump, it can either carry on evolving according to (44) if possible, or use $\widetilde{\mathcal{G}}_{10}$ to reset $q$ to 0 ; if $\hat{x}$ exits $\widehat{\mathcal{X}}$ after jump, $q$ is necessarily reset to 0 through $\widetilde{\mathcal{G}}_{10}$. In both cases, either $\hat{x}$ comes from flow and $\hat{x} \in \partial \widehat{\mathcal{X}} \subseteq \widehat{\mathcal{X}}$, or $\hat{x}$ is an output of $G$ and $\hat{x} \in \widehat{\mathcal{X}}_{d \text {,max }}$ by definition of $\widehat{\mathcal{X}}_{d, \text { max }}$. Therefore, at this point, $\hat{x} \in \widehat{\mathcal{X}}_{\max }$ and $q=0$, so that no flow is possible, and the solution necessarily jumps via $\widetilde{\mathcal{G}}_{0}$. Since $\partial \widehat{\mathcal{X}} \cap \mathcal{C}_{\varrho}=\emptyset$ according to Lemma $1, \widehat{x} \in \mathbb{R}^{n_{x}} \backslash \mathcal{C}_{\varrho}$ and $\hat{x}$ is reset to $\hat{x}+M_{d}(\tau, \hat{x})$ while $(\tau, x)$ remains unchanged and $q$ is kept to 0 . According to Lemma $5, V(\tau, x, \cdot)$ decreases through the jump, so that $\hat{x}$ is still in $\widehat{\mathcal{X}}_{\text {max }}$. Along the same lines as the proof of Theorem 2, we can show that the solution jumps a finite number of times via $\widetilde{\mathcal{G}}_{0}$, until $\hat{x} \in \mathcal{C}_{\varrho}$ and $q$ is switched back to 1 . The states $(\tau, x)$ have not changed throughout this "correction phase" and since $\mathcal{C}_{\varrho} \subset \operatorname{int}(\widehat{\mathcal{X}})$, the solution restarts evolving according to the initial observer dynamics (44). Therefore, the domain of any trajectory of (58) initialized in $\mathcal{T}_{0} \times \mathcal{X}_{0} \times \widehat{\mathcal{X}} \times\{1\}$ is composed of two parts:

- a part $\mathcal{D}_{1}$ where the system evolves according to the original observer dynamics (44) and where $\hat{x} \in \widehat{\mathcal{X}}$.

- "correction phases" $\mathcal{D}_{0}$ made of sequences of a finite number of successive jumps occurring at times where $\hat{x}$ has left (or is about to leave) $\widehat{\mathcal{X}}$. During this phase, $(\tau, x)$ is kept unchanged and the final value of $\hat{x}$ is back in $\mathcal{C}_{\varrho} \subset \widehat{\mathcal{X}}$. Therefore, $\hat{x}$ is bounded and because $\mathcal{C}_{\varrho} \subset \widehat{\mathcal{X}}$, the "correction phases" are always separated by flow or jump of the component $(\tau, x)$. This means that, after removing the trivial jumps added during the "correction phases", we recover a maximal solution $(\tau, x)$ of the plant (41). As for the Lyapunov performances, (47) is obviously preserved during the phases where the solution evolves according to (44), and $V(\tau, x, \cdot)$ is non increasing throughout the "correction phase". Therefore, the Lyapunov properties (47) are preserved along the solutions once restricted to $\mathcal{D}_{1}$.

Note that the same conclusions hold by taking $\widetilde{\mathcal{F}}=$ $\left(f_{\tau}, f, F_{m}, 0\right)$ with $F_{m}$ defined in (49a) since it does not change (47a). The only difference is that $F_{m}$ naturally forces $\hat{x}$ to remain in $\mathcal{C}_{\varrho}$ during flow, so that fewer hybrid "correction phases" are necessary.

Remark 6 In the case where $P$ is generated dynamically by Riccati/Lyapunov dynamics, the trivial jumps of $\tau$ added in (58) during the "correction phases" where $q=0$ are simply traduced by trivial jumps in the dynamics of P. In other words, $P$ is kept constant throughout the correction process.

\section{CHOICE OF DESIGN PARAMETERS}

\section{A. Choice of $\widehat{\mathcal{X}}$}

Throughout this work, $\widehat{\mathcal{X}}$ is the set in which we want/need to constrain the observer solutions. However, in practice, we have some freedom to select this set. Often, we only want the state $\hat{x}$ of the observer to remain as close as possible to the set $\mathcal{X}$ where the plant state $x$ to be estimated evolves in view of SA1. Then, the design of $\widehat{\mathcal{X}}$ and $\mathcal{C}$ satisfying SA2 consists in choosing the convex map $c$ of Lemma 5 such that $\mathcal{C}_{0}$ is as close to $\mathcal{X}$ as possible, and then select $\varrho>0$ and $\widehat{\mathcal{X}}:=\mathcal{C}_{\varrho^{\prime}}$ for some $0<\varrho<\varrho^{\prime}$.

Sets $\mathcal{C}_{0}, \mathcal{C}_{\varrho}$ and $\widehat{\mathcal{X}}$ can thus be arbitrarily close by choosing $\varrho<\varrho^{\prime}$ arbitrarily small. Nevertheless, a very small $\varrho$ may induce a very large gain $\gamma_{c}$ in the CT correction term (14) due to $\delta_{\min }$ defined in (16) being very small, or a very large number of jumps $\theta$ in the DT multi-step redesign in order to bring $c$ under the threshold $\varrho$, see (70). Therefore, a compromise must be found between constraints and numerical feasibility.

It is important to mention that although item a) of Lemma 1 imposes to take a strict inclusion $\mathcal{C}_{\varrho} \subset \widehat{\mathcal{X}}$, the observer solutions are actually shown to remain in $\mathcal{C}_{\varrho}$ in the $\mathrm{CT}$ redesign 
of Theorem 1, the multi-step DT redesign of Theorem 2 and the nominal hybrid redesign of Lemma 6 . As for the singlestep DT redesign of Lemma 4, solutions actually remain in $\mathcal{C}_{0}$. In fact, only in the hybrid redesign of Theorem 3 is the strict inclusion $\mathcal{C}_{\varrho} \subset \widehat{\mathcal{X}}$ exploited to properly define the jump logic and guarantee non-Zeno solutions.

Finally, note that, in the spirit of keeping the observer trajectories as close as possible to $\mathcal{X}$, the redesign can also be done without any explicit choice of $\widehat{\mathcal{X}}$ by:

- choosing $\gamma_{c}$ as large as numerically acceptable in the CT redesign (Table I);

- choosing the desired threshold $\varrho$ under which $c$ must be brought at each iteration in the multi-step DT redesign (Table III);

- using the single-step DT redesign (Table II) thus projecting directly into $\mathcal{C}_{0}$.

\section{B. Construction of the map $c$}

We next show how to build the map $c$ in the common practical cases where the plant solutions are known to remain in a polytope or an ellipsoid. Those typically arise when the states are known to remain within physical bounds.

Lemma 7 Suppose there exists vectors $a_{i} \in \mathbb{R}^{n_{x}}$ and scalars $b_{i}, i=1 \ldots n_{c}$, satisfying

$$
\mathcal{X} \subseteq \bigcap_{i=1}^{n_{c}}\left\{x \in \mathbb{R}^{n_{x}}: a_{i}^{\top} x \leq b_{i}\right\} \subset \widehat{\mathcal{X}} .
$$

Then the function $c: \mathbb{R}^{n_{x}} \rightarrow \mathbb{R}_{\geq 0}^{n_{c}}$ defined as

$$
\begin{aligned}
c(x) & :=\left(c_{1}(x), \ldots, c_{i}(x), \ldots, c_{n_{x}}(x)\right), \\
c_{i}(x) & :=\max \left\{a_{i}^{\top} x-b_{i}, 0\right\}^{2}
\end{aligned}
$$

is $C^{1}$ and satisfies items $a$ )-b) of Lemma 1 for some $\varrho>0$.

Proof. Consider $\mathcal{B}:=\bigcap_{i=1}^{n_{c}}\left\{x \in \mathbb{R}^{n_{x}}: a_{i}^{\top} x \leq b_{i}\right\}$, and $\overline{\mathcal{B}}:=$ $\bigcap_{i=1}^{n_{c}}\left\{x \in \mathbb{R}^{n_{x}}: a_{i}^{\top} x \leq \bar{b}_{i}\right\}$ satisfying $\mathcal{X} \subset \overline{\mathcal{B}} \subset \overline{\mathcal{B}} \subset \widehat{\mathcal{X}}$, for $\bar{b}_{i}>b_{i}$ for $i=1, \ldots, n_{c}$. Pick any point $x^{\circ} \in \mathcal{X} \subset \mathcal{B}$. By construction, it satisfies $a_{i}^{\top} x^{\circ} \leq b_{i}$ for all $i=1, \ldots, n_{c}$, so that $c_{i}\left(x^{\circ}\right)=0$ for all $i=1, \ldots, n_{c}$, and therefore $c\left(x^{\circ}\right)=0$. As this holds for any point in $\mathcal{X}$, this shows that $\mathcal{X} \subset \mathcal{C}_{0}$, with $\mathcal{C}_{0}$ defined in (1). Then, select $\varrho:=\min _{i}\left\{\bar{b}_{i}-b_{i}\right\}^{2}$. Pick any point $x \in \mathcal{C}_{\varrho}$ with $\mathcal{C}_{\varrho}$ defined in (1). Hence, we have that $\left|c_{i}(x)\right| \leq \varrho$ for any $i=1, \ldots, n_{c}$. It follows that $\left|a_{i}^{\top} x-b_{i}\right| \leq \sqrt{\varrho} \leq \bar{b}_{i}-b_{i}$ which implies $a_{i}^{\top} x \leq \bar{b}_{i}$, for all $i=1, \ldots, n_{c}$, and thus $x \in \overline{\mathcal{B}}$. Since by construction $\overline{\mathcal{B}} \subset \widehat{\mathcal{X}}$, this shows that any point in $\mathcal{C}_{\varrho}$ is also in $\widehat{\mathcal{X}}$ and prove item a). Finally, in order to show item b), it is enough to observe that the functions $c_{i}$ are quadratic outside their zeroing set, and therefore convex. This concludes the proof.

Other definitions can be considered by exploiting the shape of $\mathcal{X}$. For instance, when $\mathcal{X}$ is an ellipsoid, a scalar function $c$ can be constructed as follows.

Lemma 8 Suppose there exists a positive definite matrix $Q$ of dimension $n_{x} \times n_{x}$, a scalar $r>0$ and a vector $x_{0} \in \mathbb{R}^{n_{x}}$ satisfying $\mathcal{X} \subseteq\left\{x \in \mathbb{R}^{n_{x}}:\left(x-x_{0}\right)^{\top} Q\left(x-x_{0}\right) \leq r\right\} \subset \widehat{\mathcal{X}}$. Then, the function $c: \mathbb{R}^{n_{x}} \rightarrow \mathbb{R}_{\geq 0}, n_{c}=1$, defined as

$$
c(x):=\max \left\{\frac{\left(x-x_{0}\right)^{\top} Q\left(x-x_{0}\right)}{r}-1,0\right\}^{2} .
$$

is $C^{1}$ and verifies the items $a$ )- $b$ ) of Lemma 1 for some $\varrho>0$.

Proof. Similar to Lemma 7.

\section{EXAMPLES}

\section{A. Flow and Jump Redesign for Kalman-like Observers}

The goal of this section is to illustrate and compare the two redesign techniques (flow or jump redesign) for a continuoustime observer. Inspired by the example in [13], we consider the case in which system (3) is given by

$$
\dot{x}=A(u) x+\varphi(x), \quad y=C x
$$

where $x \in \mathbb{R}^{3}$ is the state, $C=\left(\begin{array}{lll}1 & 0 & 0\end{array}\right), A(\cdot)$ and $\varphi(\cdot)$ are defined as

$$
A(u)=\left(\begin{array}{lll}
0 & u & 0 \\
0 & 0 & u \\
0 & 0 & 0
\end{array}\right), \quad \varphi(x)=\left(\begin{array}{c}
x_{1}-\frac{1}{3} x_{1}^{3} \\
\epsilon\left(3 \sin \left(x_{1}\right)-x_{2}-\eta\right) \\
x_{3}-\frac{1}{5} x_{3}^{5}+\frac{x_{1} x_{2}}{1+x_{2}^{2}}
\end{array}\right),
$$

with $\epsilon, \eta>0$ some known parameters, and $u$ an external known input. In this example we consider the case in which there are no disturbances, i.e. we consider $d=0$ in (3). By using a quadratic-like Lyapunov function, it can be shown that SA1 is satisfied for some $\mathcal{X}_{0}, \mathcal{X}, \mathcal{U}$ and $\mathcal{D}=\emptyset$. See for instance Figure 3, in which we selected $\epsilon=0.8, \eta=5$, $x(0)=(2,-2,-2)$, and $u=\sin \left(t+\frac{\pi}{2}\right)$. From simulations, we take the following numerical values for SA1: $\mathcal{X}=\left\{x \in \mathbb{R}^{3}\right.$ : $\left.\left|x_{i}\right| \leq L_{\min }, i=1,2,3\right\}$ and $L_{\min }=8$ for an appropriate $\mathcal{X}_{0} \subset \mathcal{X}$. The set $\widehat{\mathcal{X}}$ in SA2 is selected then as $\widehat{\mathcal{X}}=\{x \in$ $\left.\mathbb{R}^{3}:\left|x_{i}\right| \leq L_{\max }, i=1,2,3\right\}$ with $L_{\max }=11$. Finally, the function $c$ of Lemma 1 is chosen as in (62) with $n_{c}=6$, and $a_{1}=(1,0,0), a_{2}=(0,1,0), a_{3}=(0,0,1), a_{4}=-a_{1}$, $a_{5}=-a_{2}, a_{6}=-a_{3}, b_{1}=b_{2}=b_{3}=b_{4}=b_{5}=b_{6}=L_{\min }$, and we choose $\varrho=1$.

Following the high-gain extended-Kalman filter design in [3], we design the observer

$$
\dot{\hat{x}}=A(u) \hat{x}+\varphi_{s}(\hat{x})+\ell D_{\ell} S(t) C^{\top}(y-C \hat{x})
$$

where $D_{\ell}=\operatorname{diag}\left(1, \ell, \ell^{2}\right)$ with $\ell \geq 1$ the high-gain parameter, $\varphi_{S}$ a function to be properly chosen, and $S(t)$ is computed as solution to the following differential Riccati equation

$$
\dot{S}=\ell\left(S A(u)^{\top}+A(u) S+Q-2 S C^{\top} C S+a S\right),
$$

where $Q$ and $S(0)$ are positive definite symmetric matrices. We have then the following result.

Proposition 1 Let $\varphi_{s}$ be any $C^{1}$ function satisfying $\mid \varphi(x)-$ $\varphi_{s}(\hat{x})|\leq \delta| x-\hat{x} \mid$ for any $(x, \hat{x}) \in \mathcal{X} \times \mathbb{R}^{n_{x}}$, for some $\delta>0$. Then, system (64) and observer (65), (66) verify Assumptions 1,2 with $P(t)=D_{\ell}^{-1} S(t)^{-1} D_{\ell}^{-1}, \beta_{c}=-(a \ell-\delta) V$. and some $\bar{\lambda}>\underline{\lambda}>0$.

Proof. The proof follows the computations given in [3] and it is omitted for space reasons. 


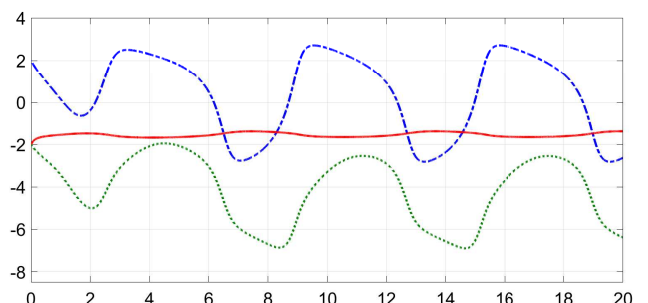

Fig. 3. Evolution of the solution to system (64) for $t \in[0,20]$ with initial conditions $x_{0}=(2,-2,-2)$.

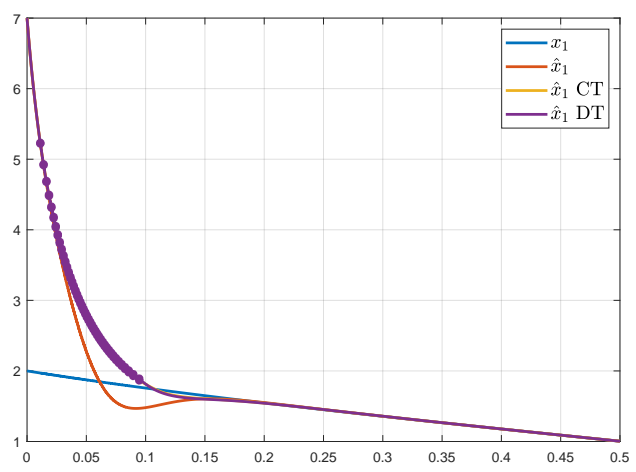

(a) $\hat{x}_{1}$

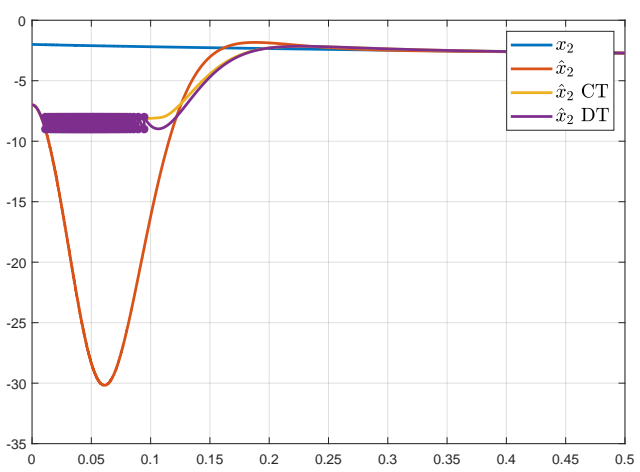

(b) $\hat{x}_{2}$

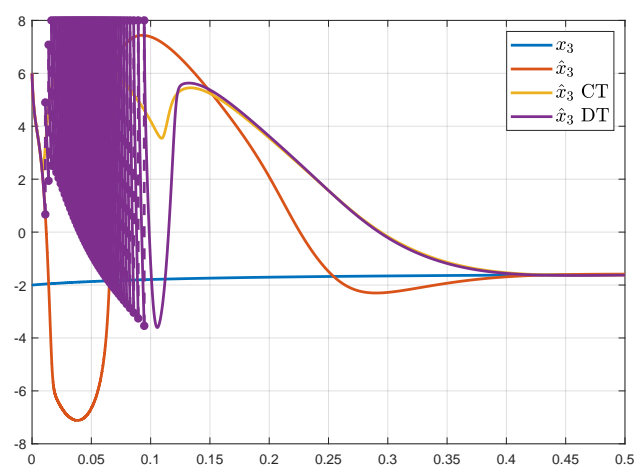

(c) $\hat{x}_{3}$

Fig. 4. Solutions of the plant (64) (blue), the nominal observer (65), (66) (red), the modified observer with flow redesign (yellow), the modified observer with jump redesign (purple), with $\hat{x}_{0}=(7,-7,6), \ell=20, a=1$.
In Figure 4, we compare the different observation strategies. With the nominal observer (65), (66), depicted in red, the peaking phenomenon [7] causes the observer state to go far away from $\mathcal{X}$ during the transient. A first solution is to apply the flow redesign tool presented in Section III (see Table I), which enables to constrain the observer state into the set $\mathcal{C}_{\varrho}$ and cut away the peaking. More precisely, we add to the observer dynamics (65) the correction term $M(t, \hat{x})$ defined in (14) with $\gamma_{c}=\ell$ and $P(t)=D_{\ell}^{-1} S(t)^{-1} D_{\ell}^{-1}$ (trajectories in yellow in Figure 4). Another solution is to apply the hybrid redesign of Section V-C (with $D=\emptyset$ ). In other words, whenever $\hat{x}$ attempts to leave $\widehat{\mathcal{X}}$, jumps are triggered to bring it back into $\widehat{\mathcal{X}}$. This can be done by successive jumps using $M_{d}$ as in Section V-C, or directly by projecting $\hat{x}$ into $\mathcal{C}_{0}$ along (54). Indeed, $\mathcal{C}_{0}$ being a polytope, this optimization problem reduces here to a QP with linear inequality constraints which can be solved on Matlab via the function quadprog. The result of simulations of this latter method appears in purple in Figure 4. Note that we have been able to use the optimization approach thanks to the polytopic form of $\widehat{\mathcal{X}}$, but in the case of a hyper-sphere, we should probably have followed the technique proposed in Theorem 2. This is not shown for space reasons, an illustration of Theorem 2 can indeed be found in [53].

Simulations confirmed the validity of the proposed approaches (flow-redesign and hybrid redesign), and suggest to actually combine both: the flow redesign keeps $\hat{x}$ as much as possible in $\widehat{\mathcal{X}}$, but if for some reason $\left(\gamma_{c}\right.$ too small or perturbations), $\hat{x}$ still attempts to leave $\widehat{\mathcal{X}}$, then the jumps act as a back-up, all this without altering the Lyapunov performances. It would be necessary to investigate in details the computational load of each method to determine which is more efficient: flow or hybrid redesign? With quadratic projections or successive jumps?

\section{B. Hybrid Redesign for the Bouncing Ball}

Consider a bouncing ball with gravity coefficient $\mathfrak{g}>0$ and restitution coefficient $0<\lambda<1$, modelled as a hybrid system with state $x=\left(x_{1}, x_{2}\right)$ representing the height and velocity of the ball, flow/jump maps

$$
f(x)=A_{c} x-\left(\begin{array}{l}
0 \\
\mathfrak{g}
\end{array}\right), \quad g(x)=A_{d} x
$$

with $A_{c}=\left(\begin{array}{ll}0 & 1 \\ 0 & 0\end{array}\right), A_{d}=\left(\begin{array}{cc}-1 & 0 \\ 0 & -\lambda\end{array}\right)$ and flow/jump sets

$$
C=\mathbb{R}_{\geq 0} \times \mathbb{R}, \quad D=\left\{\left(x_{1}, x_{2}\right) \in \mathbb{R}^{2}: x_{1}=0, x_{2} \leq 0\right\} .
$$

We assume that the height of the ball $h(x)=x_{1}=0$ is measured at the jumps only, i.e., the impacts of the ball can be detected by some sensor. No measurement is available during flows. In [33], an observer is designed for this system by adding a linear correction term in the jump dynamics, with an appropriately chosen gain, and by synchronizing the observer with the plant. This leads to the dynamics

$$
\begin{aligned}
& \left.\begin{array}{l}
\dot{x}=f(x) \\
\dot{\hat{x}}=f(\hat{x})
\end{array}\right\} x \in C, \hat{x} \in \mathbb{R}^{n_{x}}, \\
& \left.\begin{array}{l}
x^{+}=g(x) \\
\hat{x}^{+}=g(\hat{x})-L\left(\hat{x}_{1}-x_{1}\right)
\end{array}\right\} x \in D, \hat{x} \in \mathbb{R}^{n_{x}},
\end{aligned}
$$


The gain $L$ must then be chosen depending on the available knowledge about the time elapsed between successive jumps. In this particular example, since $\lambda<1$, we know that any maximal solution is Zeno, namely exists on a finite time horizon $[0, T)$ with an infinite number of jumps, i.e., the time between two successive jumps $t_{j+1}-t_{j}$ tends to 0 when $j$ tends to $+\infty$. Let us fix a bounded set $\mathcal{X}_{0}$ of initial conditions of interest, and let $\tau_{M} \geq 0$ so that $t_{j+1}-t_{j} \in\left[0, \tau_{M}\right]$ for all $j \in \mathbb{N}$ along any solution initialized in $\mathcal{X}_{0}$. According to [33], exponential convergence is ensured by choosing $L$ so that there exist $a_{c} \in \mathbb{R}$ and $a_{d}<0$, and a positive definite symmetric matrix $P \in \mathbb{R}^{n \times n}$ such that

$$
\begin{aligned}
& A_{c}^{\top} P+P A_{c} \leq a_{c} P \\
& \left(A_{d}-L_{d} H_{d}\right)^{\top} P\left(A_{d}-L_{d} H_{d}\right) \leq e^{a_{d}} P \\
& a_{c} \tau_{M}+a_{d}<0
\end{aligned}
$$

Indeed, then the Lyapunov function $V(x, \hat{x})=(\hat{x}-x)^{\top} P(\hat{x}-$ $x)$ decreases more at jumps than it increases during flow, so that $V$ decreases overall. Following [33, Example 4.2], we choose $L_{d}=(-1,-0.1487)$. The corresponding observer trajectory is plotted in Figure 5(a)-5(b) (red), and the decrease of the Lyapunov function illustrated in Figure 5(c) (blue). Convergence is indeed achieved but we observe that the height estimate becomes strongly negative during the transient, which is not physically possible. In the following, we thus redesign the observer in order to ensure the estimate remains as close as possible to the set $\mathcal{X}=[0,10] \times[-10,10]$.

The set $\mathcal{X}$ being a polytope, the function $c$ of Lemma 1 is chosen as in (62) with $n_{c}=4$, and $a_{1}=(1,0), a_{2}=(0,1)$, $a_{3}=-a_{1}, a_{4}=-a_{2}, b_{1}=b_{2}=b_{4}=10, b_{3}=0$, and $\varrho=1$. Then, $\mathcal{C}_{0}=\mathcal{X}$.

Following the hybrid redesign strategy (48)-(49), we obtain

$$
\begin{aligned}
& \left.\begin{array}{rl}
\dot{x} & =f(x) \\
\dot{\hat{x}} & =f(\hat{x})+M(\hat{x})
\end{array}\right\} x \in C, \hat{x} \in \mathbb{R}^{n_{x}}, \\
& \left.\begin{array}{l}
x^{+}=g(x) \\
\hat{x}^{+}=\psi\left(g(\hat{x})-L\left(\hat{x}_{1}-y\right)\right)
\end{array}\right\} x \in D, \hat{x} \in \mathbb{R}^{n_{x}},
\end{aligned}
$$

where $\psi: \mathbb{R}^{2} \rightarrow \mathcal{X}$ is the quadratic projection defined by

$$
\psi(\hat{x})=\operatorname{argmin}_{x \in \mathcal{X}}(\hat{x}-x)^{\top} P(\hat{x}-x)
$$

implemented with quadprog and $P=\left(\begin{array}{cc}0.2885 & -0.0012 \\ -0.0012 & 0.2733\end{array}\right)$ and $M$ is defined on $\mathbb{R}^{2}$ by $M(\hat{x})=-\gamma_{c} P^{-1} \frac{d c}{d x}(\hat{x})^{\top} c(\hat{x})$ with $\gamma_{c}=10$. Insights about the selection of $P$ are given in [33] and more extensively in [61]. Here, we do not specify a particular choice of $\widehat{\mathcal{X}}$ because our goal is to keep $\hat{x}$ close to $\mathcal{X}$ and this is achieved by taking $\gamma_{c}$ sufficiently large as explained in Section VI. The result of the simulation is provided on Figures 5(a)-5(b) (yellow). We can see that the observer actively uses the information that $x \in \mathcal{X}$ during the first interval of flow to prevent $\hat{x}$ from leaving. This practically acts like an extra measurement and enables the Lyapunov function to decrease during the transient (unlike in the original design) as illustrated on Figure 5(c) (red).

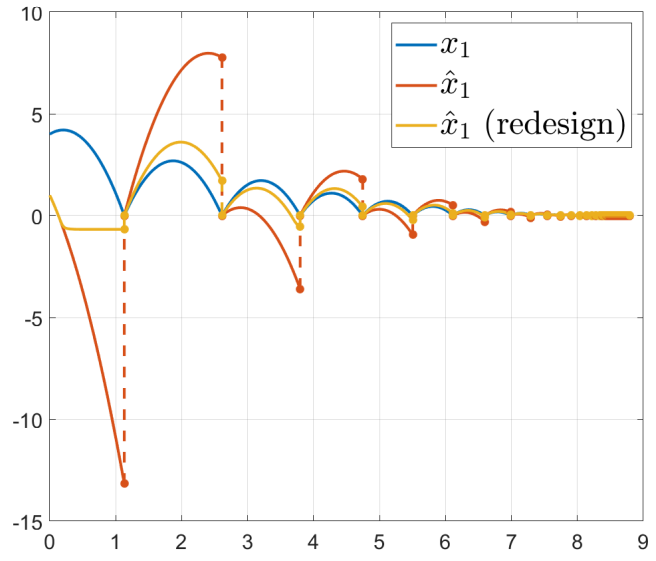

(a) $x_{1}$ : plant ; $\hat{x}_{1}$ : nominal observer ; $\hat{x}_{1}$ (redesign): redesigned observer

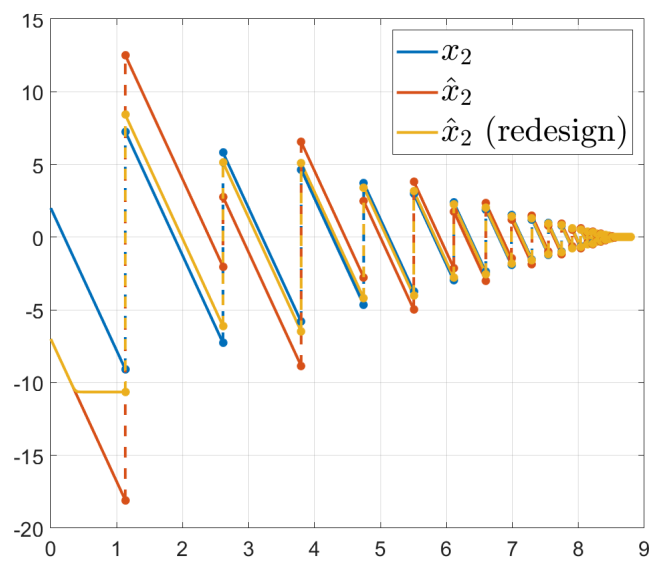

(b) $x_{2}$ : plant ; $\hat{x}_{2}$ : nominal observer ; $\hat{x}_{2}$ (redesign): redesigned observer

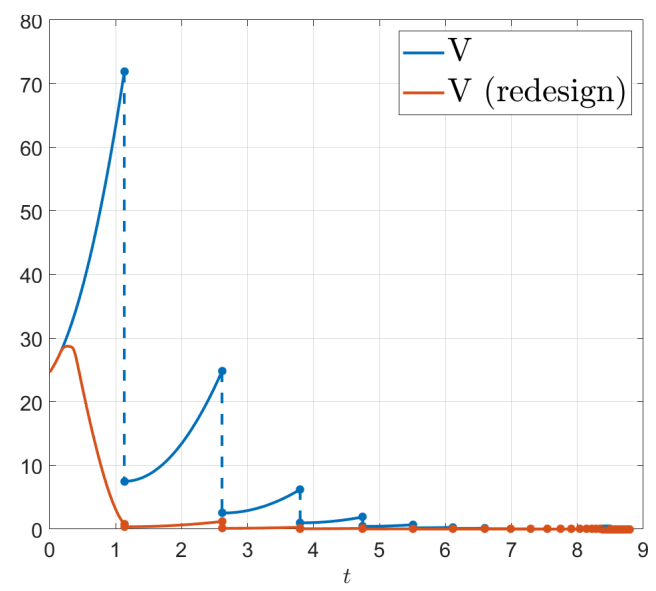

(c) $V(x, \hat{x})$ with nominal observer (blue) and modified observer (red)

Fig. 5. Solutions of the nominal observer (67), and redesigned observer (68) with $x_{0}=(4,2) \hat{x}_{0}=(1,-7)$. 


\section{CONCLUSION}

For a given dynamical system with the state evolving in a known compact set, we supposed to know an observer with performances characterized by a quadratic Lyapunov function of the error, and we addressed the problem of constraining the observer state in a desired convex set, while preserving those performances. By exploiting the knowledge of a convex function which is zero on this desired convex set, we proposed a methodology which consists in redesigning the observer's dynamics outside such a set by means of a gradient approach, and therefore avoiding the use of on-line optimization techniques. In this work we proposed a unifying point of view covering (possibly nonlinear, time-varying) continuous time, discrete time and hybrid systems that allows to use these techniques for a large number of different observers. For this, we showed how to redesign a given observer, under the same convexity assumption, in a continuous-fashion way (flow-redesign) or discrete one (jump-redesign).

The proposed methodology may find a large number of applications such as removing peaking phenomenon, avoiding numerical issues [43], [53], improving the performances in presence of large measurement noise or output feedback stabilization [41], [42]. As shown in [41]-[43], it can be also used when the coordinates of the observer and the plant's dynamics are not the same, and such change of coordinates is not globally defined. As a future work, it would be useful to quantify and compare the computational loads of the various proposed techniques.

\section{ACKNOWLEDGMENT}

We thank Laurent Praly and Maurice Heemels for the fruitful discussions, their inspiring ideas and valuable suggestions.

\section{APPENDIX}

\section{A. Proof of Theorem 2}

In light of (24), the number $v_{\max }$ defined in (39) is the largest possible value of $V_{k+1}$ after a jump with $G(k, \cdot)$ from $x_{k} \in \mathcal{X}, \hat{x}_{k} \in \widehat{\mathcal{X}}, u_{k} \in \mathcal{U}, d_{k} \in \mathcal{D}$ and for any $k \in \mathbb{N}$. Now, in light of the definition of $V_{k}$ in (22), the sets $\widehat{\mathcal{X}}_{k+1, \max }$ defined in (40) are compact elliposides, and, by using (23) and the fact that $v_{\max }$ is bounded, we conclude that $\widehat{\mathcal{X}}_{k+1, \max }$ are uniformly bounded. Therefore, there exists a compact set $\widehat{\mathcal{X}}_{\max }$ such that $\bigcup_{k \in \mathbb{N}} \widehat{\mathcal{X}}_{k+1, \max } \subseteq \widehat{\mathcal{X}}_{\max }$. With this $\widehat{\mathcal{X}}_{\max }$ so defined, let $\delta_{\max }, \gamma_{d}^{\star}$ be defined according to the proof of Lemma 5 as in (33) and (34). Finally, let $v_{\min }>0$ be defined $\mathrm{as}^{3}$

$$
\begin{aligned}
v_{\min }:=\max \left\{v \in \mathbb{R}_{\geq 0}: \forall x \in \mathcal{X}, \forall k \in \mathbb{N}\right. \\
\left.\left\{\hat{x} \in \mathbb{R}^{n_{x}}: V_{k+1}(x, \hat{x}) \leq v\right\} \subseteq \mathcal{C}_{\varrho}\right\} .
\end{aligned}
$$

with $\mathcal{C}_{\varrho}$ defined in Lemma 1 . In other words, $v_{\min }$ is the largest positive number such that the following property holds for all $k \in \mathbb{N}$

$$
\begin{aligned}
& x \in \mathcal{X} \text { and } V_{k+1}(x, \hat{x}) \leq v_{\min } \Longrightarrow \hat{x} \in \mathcal{C}_{\varrho} \subset \widehat{\mathcal{X}} . \\
& { }^{3} v_{\min } \text { is finite and positive thanks to (23) and } \mathcal{X} \text { compact. }
\end{aligned}
$$

Pick any strictly positive $\gamma_{d}$ satisfying $\gamma_{d}<\gamma_{d}^{\star}$, and with $v_{\max }, v_{\min }$ defined above, let $\theta \in \mathbb{N}$ be defined as

$$
\theta:=\operatorname{ceil}\left(\frac{\max \left\{v_{\max }-v_{\min }, 0\right\}}{\gamma_{d} \varrho^{2}}\right),
$$

with $\varrho$ given by Lemma 1 . With all the parameters so defined, the rest of the proof consists in showing that, by applying $M_{k+1}$ at most $\theta \in \mathbb{N}$ times, the value of $V_{k+1}$ is brought below the threshold $v_{\min }$. This, in turn, implies that, in light of the previous definitions, the value $G\left(k, \hat{x}_{k}, u_{k}, y_{k}\right) \in \widehat{\mathcal{X}}_{k+1, \max }$ is brought back into $\mathcal{C}_{\varrho}$ and therefore in $\widehat{\mathcal{X}} \supset \mathcal{C}_{\varrho}$ in view of item a) of Lemma 1 .

To this end, let $(k, \hat{x}) \in \mathbb{N} \times \widehat{\mathcal{X}}_{k+1, \max }$, and define $\hat{x}_{k, 1}=\hat{x}$ and $q_{k, 1}=0$. By definition of $\widehat{\mathcal{X}}_{k+1, \max }$ in (40), there exists $x_{k} \in \mathcal{X}$ such that $V_{k+1}\left(x_{k}, \hat{x}_{k, 1}\right) \leq v_{\max }$. First, let us show that for any sequence of points $\left(\hat{x}_{k, i}, q_{k, i}\right)_{i=1, \ldots, \theta+1}$ such that

$$
\left(\hat{x}_{k, i+1}, q_{k, i+1}\right) \in \widetilde{\psi}_{k+1, i}\left(\hat{x}_{k, i}, q_{k, i}\right) \quad \forall i=1 \ldots \theta
$$

we have $x_{k, i+1} \in \widehat{\mathcal{X}}_{k+1, \max }$ and

$$
V_{k+1}\left(\hat{x}_{k, i+1}, x\right) \leq V_{k+1}\left(\hat{x}_{k, i}, x\right) \quad \forall x \in \mathcal{X}
$$

for all $i=1 \ldots \theta$. From (36), we have

$$
\left(\begin{array}{l}
\hat{x}_{k, 2} \\
q_{k, 2}
\end{array}\right) \in\left(\begin{array}{c}
\hat{x}_{k, 1}+M_{k+1}\left(\hat{x}_{k, 1}\right) \\
1
\end{array}\right) \cup\left(\begin{array}{c}
\hat{x}_{k, 1} \\
0
\end{array}\right),
$$

with the second option being only possible if $\hat{x}_{k, 1} \in \mathcal{C}_{\varrho}$. By construction, $\widehat{\mathcal{X}}_{k+1, \max } \subseteq \widehat{\mathcal{X}}_{\max }$, so $\hat{x}_{k, 1} \in \widehat{\mathcal{X}}_{\max }$, and according to (32a) in Lemma 5, (72) holds in both cases for $i=1$. Besides, $V_{k+1}\left(x_{k}, \hat{x}_{k, 1}\right) \leq v_{\max }$ so that $V_{k+1}\left(x_{k}, \hat{x}_{k, 2}\right) \leq v_{\max }$ and $\hat{x}_{k, 2} \in \widehat{\mathcal{X}}_{k+1, \max }$. Iterating and using the same arguments recursively, we obtain that $\hat{x}_{k, i} \in \widehat{\mathcal{X}}_{k+1, \max }$ for all $i=1, \ldots, \theta+1$ and that for all $x \in \mathcal{X}, V_{k+1}\left(x, \hat{x}_{k, j+1}\right) \leq V_{k+1}\left(x, \hat{x}_{k, j}\right), j=1, \ldots, \theta$, which gives $V_{k+1}\left(x, \hat{x}_{k, \theta+1}\right) \leq V_{k+1}(x, \hat{x})$. Observing that for any $z \in \psi_{k+1}(\hat{x})$, there exists a sequence $\left(\hat{x}_{k, i}, q_{k, i}\right)_{i=1, \ldots, \theta+1}$ verifying (71) with $q_{k, 1}=0$ and $z=\hat{x}_{k, \theta+1}$, we deduce that (28a) holds with $\mathcal{S}_{k+1}=\widehat{\mathcal{X}}_{k+1, \text { max }}$.

In order to prove (28b), recall that at the first step, $q_{k, 1}$ is initialized at 0 , and after that, we compute $\left(\hat{x}_{k, i+1}, q_{k, i+1}\right)$ according to (36). From this definition, we can see that either $q_{k, i}=0$ for all $i=1 \ldots \theta+1$, or there exists $1 \leq \theta_{k} \leq \theta$ such that $q_{k, i}=0$ for all $i=1 \ldots \theta_{k}$, and $q_{k, i}=1$ for all $i=\theta_{k}+1 \ldots \theta+1$. In the latter case, still from the definition of $\tilde{\psi}_{k+1}$, it means that $\hat{x}_{k, \theta+1}=\hat{x}_{k, \theta_{k}} \in \mathcal{C}_{\varrho}$, and by recalling item a) in Lemma 1 , we obtain $\hat{x}_{k, \theta+1} \in \widehat{\mathcal{X}}$. Now suppose that such $\theta_{k}$ does not exists, namely, $q_{k, i}=0$ for all $i=1 \ldots \theta+1$. From (36), this means that for all $i=1, \ldots, \theta$, $\hat{x}_{k, i} \in \mathbb{R}^{n_{x}} \backslash \operatorname{int}\left(\mathcal{C}_{\varrho}\right)$, and $\hat{x}_{k, i+1}=\hat{x}_{k, i}+M_{k+1}\left(\hat{x}_{k, i}\right.$. As a consequence, we can use inequality (32b) for each $i=1, \ldots, \theta$, obtaining $V_{k+1}\left(x_{k}, \hat{x}_{k, \theta+1}\right) \leq v_{\max }-\theta \gamma_{d} \varrho^{2} \leq v_{\min }$, by the definition of $\theta$ in (31). Since $x_{k} \in \mathcal{X}$, using (69) and item a) of Lemma 1 , gives $\hat{x}_{k, \theta+1} \in \mathcal{C}_{\varrho} \subset \widehat{\mathcal{X}}$. This implies that $\widetilde{\psi}_{k+1}\left(\widehat{\mathcal{X}}_{k+1, \max } \times\{0\}\right) \subseteq \widehat{\mathcal{X}} \times\{0,1\}$, and therefore, $\psi_{k+1}\left(\widehat{\mathcal{X}}_{k+1, \max }\right) \subseteq \widehat{\mathcal{X}}$ which is (28b). As for (28c), it holds by construction and Assumption 3 . 


\section{REFERENCES}

[1] R. Kalman, "A new approach to linear filtering and prediction problems," Journal of basic Engineering, vol. 82, no. 1, pp. 35-45, 1960.

[2] G. Besançon, G. Bornard, and H. Hammouri, "Observer synthesis for a class of nonlinear control systems," European Journal of control, vol. 2, no. 3, pp. 176-192, 1996

[3] G. Besançon, "Further results on high gain observers for nonlinear systems," in 38th IEEE Conference on Decision and Control, vol. 3, 1999, pp. 2904-2909.

[4] F. Luenberger, "An introduction to observers," IEEE Transactions on automatic control, vol. 16, no. 6, pp. 596-602, 1971.

[5] A. Zemouche and M. Boutayeb, "On LMI conditions to design observers for lipschitz nonlinear systems," Automatica, vol. 49, no. 2, pp. 585-591, 2013.

[6] M. Arcak and P. Kokotović, "Nonlinear observers: a circle criterion design and robustness analysis," Automatica, vol. 37, no. 12, pp. $1923-$ 1930,2001

[7] H. Khalil and L. Praly, "High-gain observers in nonlinear feedback control," International Journal of Robust and Nonlinear Control, vol. 24, no. 6, pp. 993-1015, 2014.

[8] D. Astolfi and L. Marconi, "A high-gain nonlinear observer with limited gain power," IEEE Transactions on Automatic Control, vol. 60, no. 11, pp. 3059-3064, 2015.

[9] V. Andrieu and L. Praly, "On the existence of a kazantziskravaris/luenberger observer," SIAM Journal on Control and Optimization, vol. 45, no. 2, pp. 432-456, 2006.

[10] P. Jouan, "Immersion of nonlinear systems into linear systems modulo output injection," SIAM Journal on Control and Optimization, vol. 41, no. 6, pp. 1756-1778, 2003.

[11] G. Besançon and A. Ticlea, "An immersion-based observer design for rank-observable nonlinear systems," IEEE Transactions on Automatic Control, vol. 52, no. 1, pp. 83-88, 2007.

[12] P. Bernard, L. Praly, and V. Andrieu, "Expressing an observer in preferred coordinates by transforming an injective immersion into a surjective diffeomorphism," SIAM Journal on Control and Optimization, vol. 56, no. 3, pp. 2327-2352, 2018.

[13] N. Boizot, E. Busvelle, and J. Gauthier, "An adaptive high-gain observer for nonlinear systems," Automatica, vol. 46, no. 9, pp. 1483-1488, 2010.

[14] D. Astolfi, A. Alessandri, and L. Zaccarian, "Stubborn and dead-zone redesign for nonlinear observers and filters," IEEE Transactions on Automatic Control, vol. 66, no. 2, pp. 667-682, 2021.

[15] C. Califano, S. Monaco, and D. Normand-Cyrot, "On the observer design in discrete-time," Systems \& Control Letters, vol. 49, no. 4, pp. 255-265, 2003.

[16] G. Ciccarella, M. Dalla Mora, and A. Germani, "Observers for discretetime nonlinear systems," Systems \& Control Letters, vol. 20, no. 5, pp. 373-382, 1993.

[17] S. Ibrir, "Circle-criterion approach to discrete-time nonlinear observer design," Automatica, vol. 43, no. 8, pp. 1432-1441, 2007.

[18] L. Xie, Y. C. Soh, and C. De Souza, "Robust kalman filtering for uncertain discrete-time systems," IEEE Transactions on Automatic Control, vol. 39, no. 6, pp. 1310-1314, 1994.

[19] A. Zemouche and M. Boutayeb, "Comments on a note on observers for discrete-time lipschitz nonlinear systems," IEEE Transactions on Circuits and Systems II: Express Briefs, vol. 60, no. 1, 2013.

[20] M. Boutayeb, H. Rafaralahy, and M. Darouach, "Convergence analysis of the extended kalman filter used as an observer for nonlinear deterministic discrete-time systems," IEEE Transactions on Automatic Control, vol. 42, no. 4, pp. 581-586, 1997.

[21] L. Brivadis, V. Andrieu, and U. Serres, "Luenberger observers for discrete-time nonlinear systems," in IEEE 58th Conference on Decision and Control, 2019, pp. 3435-3440.

[22] R. Goebel, R. G. Sanfelice, and A. R. Teel, Hybrid Dynamical Systems: modeling, stability, and robustness. Princeton University Press, 2012.

[23] D. Astolfi, R. Postoyan, and D. Nesic, "Uniting observers," IEEE Transactions on Automatic Control, vol. 65, no. 7, pp. 2867-2882, 2020.

[24] T. Raff and F. Allgöwer, "Observers with impulsive dynamical behavior for linear and nonlinear continuous-time systems," IEEE 46th Conference on Decision and Control, pp. 4287-4292, 2007.

[25] F. Ferrante, F. Gouaisbaut, R. G. Sanfelice, and S. Tarbouriech, "State estimation of linear systems in the presence of sporadic measurements," Automatica, vol. 73, pp. 101-109, 2016.

[26] R. Postoyan and D. Nešić, "A framework for the observer design for networked control systems," IEEE Transactions on Automatic Control, vol. 57, no. 5, pp. 1309-1314, 2011.
[27] I. Karafyllis and C. Kravaris, "From continuous-time design to sampleddata design of observers," IEEE Transactions on Automatic Control, vol. 54, no. 9, pp. 2169-2174, 2009.

[28] T. Dinh, V. Andrieu, M. Nadri, and U. Serres, "Continuous-discrete time observer design for lipschitz systems with sampled measurements," IEEE Transactions on Automatic Control, vol. 60, no. 3, pp. 787-792, 2014.

[29] A. Alessandri and P. Coletta, "Switching observers for continuous-time and discrete-time linear systems," American Control Conference, pp. 2516-2521, 2001.

[30] S. Petterson, "Designing switched observers for switched systems using multiple Lyapunov functions and dwell-time switching," IFAC Conference on Analysis and Design of Hybrid Systems, 2006.

[31] A. Tanwani, H. Shim, and D. Liberzon, "Observability for switched linear systems: characterization and observer design," IEEE Transactions on Automatic Control, vol. 58, no. 4, pp. 891-904, 2012.

[32] E. A. Medina and D. A. Lawrence, "State estimation for linear impulsive systems," American Control Conference, pp. 1183-1188, 2009.

[33] P. Bernard and R. Sanfelice, "Observers for hybrid systems with linear maps and known jump times," IEEE 57th Conference on Decision and Control, 2018.

[34] H. Ríos and A. Teel, "A hybrid fixed-time observer for state estimation of linear systems," Automatica, vol. 87, pp. 103-112, 2018.

[35] Y. Li and R. Sanfelice, "A finite-time convergent observer with robustness to piecewise-constant measurement noise," Automatica, vol. 57, pp. 222-230, 2015.

[36] V. Andrieu, C. Prieur, S. Tarbouriech, and L. Zaccarian, "A hybrid scheme for reducing peaking in high-gain observers for a class of nonlinear systems," Automatica, vol. 72, pp. 138-146, 2016.

[37] C. Rao, J. Rawlings, and D. Mayne, "Constrained state estimation for nonlinear discrete-time systems: Stability and moving horizon approximations," IEEE Transactions on Automatic Control, vol. 48, no. 2, pp. 246-258, 2003.

[38] P. Blondel, R. Postoyan, S. Raël, S. Benjamin, and P. Desprez, "Nonlinear circle-criterion observer design for an electrochemical battery model," IEEE Transactions on Control Systems Technology, vol. 27, no. 2, pp. 889-897, 2019.

[39] L. Farina and S. Rinaldi, Positive linear systems: theory and applications. John Wiley \& Sons, 2011, vol. 50

[40] M. Maggiore and K. Passino, "A separation principle for a class of nonuco systems," IEEE Transactions on Automatic Control, vol. 48, no. 7, pp. 1122-1133, 2003.

[41] D. Astolfi and L. Praly, "Output feedback stabilization for siso nonlinear systems with an observer in the original coordinates," in 52nd IEEE Conference on Decision and Control, 2013, pp. 5927-5932.

[42] — , "Integral action in output feedback for multi-input multi-output nonlinear systems," IEEE Transactions on Automatic Control, vol. 62, no. 4, pp. 1559-1574, 2017.

[43] P. Bernard and L. Marconi, "Hybrid implementation of observers in plant's coordinates with a finite number of approximate inversions and global convergence," Automatica, vol. 111, p. 108654, 2020.

[44] D. Simon and T. Chia, "Kalman filtering with state equality constraints," IEEE Transactions on Aerospace and Electronic Systems, vol. 38, no. 1, pp. 128-136, 2002.

[45] A. D. Ames, X. Xu, J. W. Grizzle, and P. Tabuada, "Control barrier function based quadratic programs for safety critical systems," IEEE Transactions on Automatic Control, vol. 62, no. 8, pp. 3861-3876, 2016.

[46] A. Tanwani, B. Brogliato, and C. Prieur, "Stability and observer design for lur'e systems with multivalued, nonmonotone, time-varying nonlinearities and state jumps," SIAM Journal on Control and Optimization, vol. 52, no. 6, pp. 3639-3672, 2014.

[47] W. Heemels, M. Camlibel, and M. Heertjes, "Oblique projected dynamical systems and incremental stability under state constraints," IEEE Control Systems Letters, vol. 4, no. 4, pp. 1060-1065, 2020.

[48] S. Ko and R. R. Bitmead, "State estimation for linear systems with state equality constraints," Automatica, vol. 43, pp. 1363-1368, 2007.

[49] N. Gupta, "Kalman filtering in the presence of state space equality constraints," Chinese Control Conference, 2007.

[50] C. Yang and E. Blasch, "Kalman filtering with nonlinear state constraints," IEEE Transactions on Aerospace and Electronic Systems, vol. 45, no. 1, pp. 70-84, 2009.

[51] D. Simon, "Kalman filtering with state constraints: a survey of linear and nonlinear algorithms," IET Control Theory \& Applications, vol. 4, no. 8, pp. 1303-1318, 2010.

[52] S. Berkane, A. Tayebi, and A. R. Teel, "Hybrid constrained estimation for linear time-varying systems," 57th IEEE Conference on Decision and Control, 2018. 
[53] D. Astolfi, P. Bernard, R. Postoyan, and L. Marconi, "Redesign of discrete-time nonlinear observers with state estimate constraints guaranteed in prescribed convex set," in 11th IFAC Nonlinear Control System Symposium, 2019.

[54] R. G. Sanfelice and L. Praly, "Convergence of nonlinear observers on $\mathbb{R}^{n}$ with a riemannian metric (part i)," IEEE Transactions on Automatic Control, vol. 57, no. 7, pp. 1709-1722, 2012.

[55] E. M. Bronstein, "Approximation of convex sets by polytopes," Journal of Mathematical Science, vol. 153, no. 6, pp. 727-762, 2008

[56] J. Gauthier, H. Hammouri, and S. Othman, "A simple observer for nonlinear systems application to bioreactors," IEEE Transactions on Automatic Control, vol. 37, no. 6, pp. 875-880, 1992.

[57] A. Bemporad, M. H. Heemels, and B. D. Schutter, "On hybrid systems and closed-loop mpc systems," IEEE Transactions on Automatic Control, vol. 47, no. 5, pp. 863-869, 2002.

[58] A. Bemporad, M. Morari, V. Dua, and E. N. Pistikopoulos, "The explicit linear quadratic regulator for constrained systems," Automatica, vol. 38 , pp. 3-20, 2002.

[59] C. Cai and A. R. Teel, "Characterizations of input-to-state stability for hybrid systems," Systems \& Control Letters, vol. 58, pp. 47-53, 2009.

[60] R. Postoyan, N. van de Wouw, D. Nesic, and W. Heemels, "Tracking control fornonlinear networked control systems," IEEE Transactions on Automatic Control, vol. 59, no. 6, pp. 1539-1554, 2014.

[61] P. Bernard and R. Sanfelice, "Robust observer design for hybrid dynamical systems with linear maps and approximately known jump times," Automatica, 2019. [Online]. Available: HAL https://hal.archivesouvertes.fr/hal-02187411

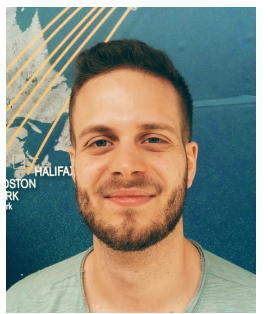

Daniele Astolfi received the B.S. and M.S. degrees in automation engineering from the University of Bologna, Italy, in 2009 and 2012, respectively. He obtained a joint Ph.D. degree in Control Theory from the University of Bologna, Italy, and from Mines ParisTech, France, in 2016. For his works on observer design and output regulation, he obtained the 2016 Best Italian Ph.D. Thesis Award in Control given by SIDRA. In 2016 and 2017, he has been a Research Assistant at the University of Lorraine (CRAN), Nancy, France. Since 2018, he is a CNRS Researcher at LAGEPP, Lyon, France. His research interests include observer design, feedback stabilization and output regulation for nonlinear systems, networked control systems, hybrid systems, and multi-agent systems. $\mathrm{He}$ serves as an associate editor of the journal Automatica since 2018.

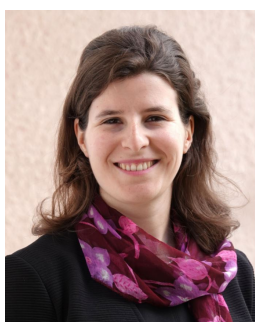

Pauline Bernard graduated in Applied Mathematics from MINES ParisTech in 2014. She joined the Systems and Control Center of MINES ParisTech and obtained her Ph.D. in Mathematics and Control from PSL University in 2017. For her work on Observer design for nonlinear systems, she obtained the European Ph.D. award on Control for Complex and Heterogeneous Systems 2018. As a post-doctoral scholar, she then visited the Hybrid Systems Lab at the University California Santa Cruz, USA, and the Center for Research on Complex Automated Systems at the University of Bologna, Italy. In 2019, she became an assistant professor at the Systems and Control Center of MINES ParisTech, PSL University, France. Her research interests cover the observation and output regulation of nonlinear and hybrid systems.

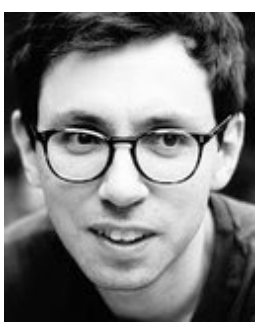

Romain Postoyan received the "Ingénieur" degree in Electrical and Control Engineering from ENSEEIHT (France) in 2005. He obtained the M.Sc. by Research in Control Theory \& Application from Coventry University (United Kingdom) in 2006 and the Ph.D. in Control Theory from Université Paris-Sud (France) in 2009. In 2010, he was a research assistant at the University of Melbourne (Australia). Since 2011, he is a CNRS researcher at the "Centre de Recherche en Automatique de Nancy" (France). He received the "Habilitation à Diriger des Recherches (HDR)" in 2019 from Université de Lorraine (Nancy, France). He serves/served as an associate editor for the journals: Automatica, IEEE Control Systems Letters and IMA Journal of Mathematical Control and Information.

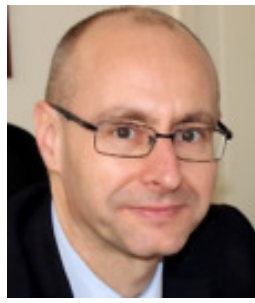

Lorenzo Marconi graduated in 1995 in electrical engineering from the University of Bologna. Since 1995 he has been with the Department of Electronics, Computer Science and Systems at University of Bologna, where he obtained his Ph.D. degree in 1998. From 1999 he has been an assistant professor in the same department where he is now a full professor. He has held visiting positions at various academic/research international institutions. He is a co-author of more than 250 technical publications on the subject of linear and nonlinear feedback designs published on international journals, books and conference proceedings. In 2005, he received the "Outstanding Application Paper Award" from IFAC for a coauthored paper published on Automatica. He is also the co-recipient of the 2014 IEEE Control Systems Magazine Outstanding Paper Award for the best paper published in the magazine in the period 2012-2013 and of the 2018 O. Hugo Schuck Best Paper Award. He is Fellow of IEEE for contributions to feedback design of nonlinear systems and unmanned aerial vehicles. His current research interests include nonlinear control, output regulation, control of autonomous vehicles, fault detection and isolation, and fault tolerant control. 